\title{
UNIFIED DUALITY FOR VECTOR OPTIMIZATION PROBLEM OVER CONES INVOLVING SUPPORT FUNCTIONS
}

\author{
Surjeet Kaur Suneja ${ }^{1}$ And Pooja Louhan ${ }^{2}$
}

\begin{abstract}
In this paper we give necessary and sufficient optimality conditions for a vector optimization problem over cones involving support functions in objective as well as constraints, using cone-convex and other related functions. We also associate a unified dual to the primal problem and establish weak, strong and converse duality results. A number of previously studied problems appear as special cases.
\end{abstract}

Keywords. Vector optimization, cones, support function, optimality, duality.

Mathematics Subject Classification. 90C29, 90C46, 90C25, $90 \mathrm{C} 26$.

\section{INTRODUCTION}

Vector Optimization Problems are those in which we have more than one objective function. In vector optimization one investigates optimal points such as weak minimum, minimum, proper minimum and strong minimum of a nonempty subset of a partially ordered linear space. When the ordering of the partially ordered linear space is done through cones the problem is referred to as vector optimization problem over cones. Problems of this type can be found not only in mathematics but also in engineering and economics. Convexity plays a key role in the theory of vector optimization. It turns out to be a powerful tool for the

Received May 5, 2013. Accepted December 3, 2013.

1 Department of Mathematics, Miranda House, University of Delhi, Delhi-110 007, India. surjeetsuneja@gmail.com

2 Department of Mathematics, University of Delhi, Delhi-110 007, India. poojalouhan@gmail.com. 
investigation of vector optimization problems in a partially ordered linear space for two main reasons. Firstly, separation theorems due to convexity are especially helpful for the development of Lagrangian theory and secondly, a partial ordering in a real linear space can be characterized by a convex cone and therefore theorems concerning convex cones are very useful. Weir et al. [18] gave the definition of cone-convex function. Luc [3] used cone-convex and cone-quasiconvex functions to provide a detailed theory on vector optimization problems. Cambini [13] introduced several classes of concave vector-valued functions which are possible extensions of scalar generalized concavity using the order relations generated by a cone or the interior of a cone or a cone without origin. Suneja et al. [16] defined second-order cone-convex, pseudoconvex, strongly pseudoconvex and quasiconvex functions. Flores-Bazán and Vera [4] presented a unified approach for dealing with both the notions of efficiency and weak efficiency (described in terms of a preference relation determined by a closed convex cone) under generalized quasiconvexity assumptions. Jahn [10] introduced the various definitions of generalized quasiconvex and pseudoconvex maps and established optimality conditions and duality results for a given vector optimization problem. Recently Suneja et al. [15] gave the definitions of various types of cone-pseudoconvex and cone-quasiconvex functions and studied the relations between them.

Support functions play an important role in convex analysis and optimization. In fact they help in characterizing a closed convex set and the position of a point relative to a set with powerful analytical tools. This allows one to translate problem on convex sets to a problem in terms of convex functions. The presence of support functions in any optimization problem makes the problem nondifferentiable and hence we have to deal with subdifferentials. So the main reason to use support functions for studying nondifferentiable optimization problems is that their subdifferentials are very well known. Various researchers have studied optimization problems involving support functions. Schechter $[11,12]$ established optimality and duality results for a nonlinear problem involving support functions in the objective. Husain et al. [9] derived optimality conditions for a nonlinear program in which a support function appears in the objective as well as in each constraint function. They presented Wolfe and Mond-Weir type duals to this program and established various duality results under suitable convexity and generalized convexity assumptions. Recently Husain et al. [8] studied a multiobjective programming problem containing support functions. They constructed Wolfe and Mond-Weir type duals to this problem and validated various duality results under invexity and generalized invexity assumptions.

We moved a step ahead with this paper and considered a vector optimization problem over cones which includes support functions of compact convex sets in objective as well as in constraints. It is also shown in the paper that, under particular conditions, our problem reduces to the problems considered by various researchers (see $[8,9,11])$. On the lines of Craven [1], we have given necessary optimality conditions for a point to be a weak minimum of our vector optimization problem and then using the constraint qualification given by Suneja et al. [15] we prove 
KKT type necessary optimality conditions. We have also established sufficient optimality conditions using cone-convex, (strictly, strongly) cone-pseudoconvex and cone-quasiconvex functions.

The motivation behind the study of such problems arises from the fact that even though the objective function and/or constraint function of the primal problem are nondifferentiable, we can always associate dual problems to it which are differentiable. Generally it is easier to solve a differentiable problem than to solve a nondifferentiable problem. Using this fact, we have associated a unified dual with the primal problem and established weak, strong and converse duality results under cone-convexity, cone-pseudoconvexity and cone-quasiconvexity assumptions. In converse duality, we start from the optimal solution of dual problem and under suitable assumptions we arrive at the optimal solution of the primal problem. As mentioned earlier the dual problem is easier to solve as compared to the primal problem, so we can find the optimal solution for dual and using converse duality we can compute the optimal solution for primal. Finally, we have related our primal and dual problems with special cases that often occur in the literature in which a support function becomes the square root of a positive semi-definite quadratic form or an $L_{p}$ norm by suitably defining the compact convex sets.

We can find many problems of facility location and portfolio selection modeled as vector optimization problem which reflect the utility of our problem.

\section{Notations AND DEFinitions}

Let $K \subseteq \mathbb{R}^{m}$ be a closed convex pointed $(K \cap(-K)=\{0\})$ cone such that $\operatorname{int} K \neq \phi$, where int $K$ denotes the interior of $K$. The positive dual cone $K^{+}$and strict positive dual cone $K^{s+}$ of $K$ are defined as follows:

$$
\begin{gathered}
K^{+}=\left\{\lambda \in \mathbb{R}^{m}: \lambda^{T} x \geq 0, \quad \text { for all } x \in K\right\}, \\
K^{s+}=\left\{\lambda \in \mathbb{R}^{m}: \lambda^{T} x>0, \quad \text { for all } x \in K \backslash\{0\}\right\} .
\end{gathered}
$$

The closedness and convexity of the cone $K$ is equivalent to $K=\left(K^{+}\right)^{+}$by the bipolar theorem. In this case,

$$
x \in K \Longleftrightarrow \lambda^{T} x \geq 0 \quad \forall \lambda \in K^{+} .
$$

Moreover, by [5] we have

$$
x \in \operatorname{int} K \Longleftrightarrow \lambda^{T} x>0 \quad \forall \lambda \in K^{+} \backslash\{0\} .
$$

In this section, we recall some of the basic definitions and results, which are to be used throughout the paper.

Let $f: \mathbb{R}^{n} \longrightarrow \mathbb{R}^{m}$, where $f=\left(f_{1}, f_{2}, \ldots, f_{m}\right)^{T}$ and $h: \mathbb{R}^{n} \longrightarrow \mathbb{R}$. 
Definition 2.1. The function $f$ is said to be $K$-convex at $\bar{x} \in \mathbb{R}^{n}$, if for all $x \in \mathbb{R}^{n}$ and $t \in[0,1]$,

$$
t f(x)+(1-t) f(\bar{x})-f(t x+(1-t) \bar{x}) \in K .
$$

The function $f$ is said to be $K$-convex if it is $K$-convex at each $\bar{x} \in \mathbb{R}^{n}$.

Remark 2.2 [13]. If $f$ is a differentiable function, then $f$ is $K$-convex if and only if for all $x, \bar{x} \in \mathbb{R}^{n}$,

$$
f(x)-f(\bar{x})-\nabla f(\bar{x})(x-\bar{x}) \in K
$$

where $\nabla f(\bar{x})=\left[\nabla f_{1}(\bar{x}), \nabla f_{2}(\bar{x}), \ldots, \nabla f_{m}(\bar{x})\right]^{T}$ is the $m \times n$ Jacobian matrix of $f$ at $\bar{x}$ and for each $i=1,2, \ldots, m, \nabla f_{i}(\bar{x})=\left(\frac{\partial f_{i}}{\partial x_{1}}, \frac{\partial f_{i}}{\partial x_{2}}, \ldots, \frac{\partial f_{i}}{\partial x_{n}}\right)^{T}$ is the $n \times 1$ Gradient vector of $f_{i}$ at $\bar{x}$.

Definition 2.3. The function $f$ is said to be $K$-pseudoconvex at $\bar{x} \in \mathbb{R}^{n}$, if for every $x \in \mathbb{R}^{n}$,

$$
-\nabla f(\bar{x})(x-\bar{x}) \notin \operatorname{int} K \quad \Rightarrow \quad-[f(x)-f(\bar{x})] \notin \operatorname{int} K .
$$

Remark 2.4. If we replace $f$ by $-f$ and $\bar{x}$ by any $y \in \mathbb{R}^{n}$, then above definition reduces to the definition of (int $K$, int $K$ )-pseudoconcave function given by Cambini [13] where $S=\mathbb{R}^{n}$.

On the lines of Suneja et al. [15] we give the following definition.

Definition 2.5. The function $f$ is said to be strictly $K$-pseudoconvex at $\bar{x} \in \mathbb{R}^{n}$, if for every $x \in \mathbb{R}^{n}$,

$$
-\nabla f(\bar{x})(x-\bar{x}) \notin \operatorname{int} K \quad \Rightarrow \quad-[f(x)-f(\bar{x})] \notin K \backslash\{0\} .
$$

Remark 2.6. If all the conditions of Remark 2.4 are satisfied then the above definition reduces to the definition of $(K \backslash\{0\}$, int $K)$-pseudoconcave function given by Cambini [13].

Remark 2.7. Every strictly $K$-pseudoconvex function at $\bar{x}$ is $K$-pseudoconvex at $\bar{x}$.

Definition 2.8. The function $f$ is said to be strongly $K$-pseudoconvex at $\bar{x} \in \mathbb{R}^{n}$, if for every $x \in \mathbb{R}^{n}$,

$$
-\nabla f(\bar{x})(x-\bar{x}) \notin \operatorname{int} K \quad \Rightarrow \quad f(x)-f(\bar{x}) \in K .
$$

Remark 2.9. Every strongly $K$-pseudoconvex function at $\bar{x}$ is strictly $K$-pseudoconvex and hence $K$-pseudoconvex at $\bar{x}$.

Definition 2.10. The function $f$ is said to be $K$-quasiconvex at $\bar{x} \in \mathbb{R}^{n}$, if for every $x \in \mathbb{R}^{n}$,

$$
f(x)-f(\bar{x}) \notin \operatorname{int} K \quad \Rightarrow \quad-\nabla f(\bar{x})(x-\bar{x}) \in K .
$$


Now we briefly describe the Clarke's [6] notion of generalized directional derivative and subdifferential of a locally Lipschitz function.

The function $h$ is said to be locally Lipschitz at $\bar{x} \in \mathbb{R}^{n}$ if there exists a nonnegative constant $L$ and a neighborhood $N(\bar{x})$ of $\bar{x}$ such that for all $x, y \in N(\bar{x})$, we have

$$
\|h(x)-h(y)\| \leq L\|x-y\| .
$$

The vector-valued function $f$ is locally Lipschitz at $\bar{x} \in \mathbb{R}^{n}$ if for each $i=$ $1,2, \ldots, m, f_{i}$ is locally Lipschitz at $\bar{x} \in \mathbb{R}^{n}$.

The Clarke generalized directional derivative of a locally Lipschitz function $h$ at $\bar{x} \in \mathbb{R}^{n}$ in the direction $d \in \mathbb{R}^{n}$ is given as

$$
h^{\circ}(\bar{x} ; d)=\limsup _{y \rightarrow \bar{x}, t \rightarrow 0^{+}} \frac{h(y+t d)-h(y)}{t},
$$

where $y \in \mathbb{R}^{n}$ and $t>0$.

The Clarke generalized gradient or the Clarke subdifferential of a locally Lipschitz function $h$ at $\bar{x} \in \mathbb{R}^{n}$ is given as

$$
\partial^{c} h(\bar{x})=\left\{\xi \in \mathbb{R}^{n}: h^{\circ}(\bar{x}, d) \geq \xi^{T} d, \text { for all } d \in \mathbb{R}^{n}\right\} .
$$

If $h$ is convex function, then for any $\bar{x} \in \mathbb{R}^{n}, h$ is locally Lipschitz at $\bar{x}$ and in this case

$$
\partial^{c} h(\bar{x})=\partial h(\bar{x})=\left\{\xi \in \mathbb{R}^{n}: h(x)-h(\bar{x}) \geq(x-\bar{x})^{T} \xi, \text { for all } x \in \mathbb{R}^{n}\right\} .
$$

Also, if $h$ is continuously differentiable at $\bar{x}$, then $h$ is locally Lipschitz at $\bar{x}$ and $\partial^{c} h(\bar{x})=\{\nabla h(\bar{x})\}$.

We also review some well known facts about support functions. Let $C \subseteq \mathbb{R}^{n}$ be a compact convex set. The support function of $C$ is defined by

$$
s(x \mid C)=\max _{z \in C} x^{T} z .
$$

The support function of a compact convex set, being convex and finite everywhere, has a subgradient at every $\bar{x} \in \mathbb{R}^{n}$ and the set of all subgradients at $\bar{x}$, that is, the subdifferential at $\bar{x}$ is given by

$$
\partial s(\bar{x} \mid C)=\left\{z \in C: \bar{x}^{T} z=s(\bar{x} \mid C)\right\} .
$$

\section{Optimality CONDitions}

Consider the vector optimization problem (NVP) K-Minimize

$$
f(x)+s(x \mid C) k=\left(f_{1}(x)+s(x \mid C) k_{1}, f_{2}(x)+s(x \mid C) k_{2}, \ldots, f_{m}(x)+s(x \mid C) k_{m}\right)^{T}
$$


subject to

$$
\begin{aligned}
-g(x)-s(x \mid D) q= & -\left(g_{1}(x)+s(x \mid D) q_{1}, g_{2}(x)\right. \\
& \left.+s(x \mid D) q_{2}, \ldots, g_{p}(x)+s(x \mid D) q_{p}\right)^{T} \in Q,
\end{aligned}
$$

where $f: \mathbb{R}^{n} \longrightarrow \mathbb{R}^{m}$ and $g: \mathbb{R}^{n} \longrightarrow \mathbb{R}^{p}$ are continuously differentiable vector-valued functions, $C$ and $D$ are non-empty compact convex subsets of $\mathbb{R}^{n}$, $K$ and $Q$ are closed convex pointed cones in $\mathbb{R}^{m}$ and $\mathbb{R}^{p}$ respectively with nonempty interiors and $k=\left(k_{1}, k_{2}, \ldots, k_{m}\right)^{T} \in \operatorname{int} K$ and $q=\left(q_{1}, q_{2}, \ldots, q_{p}\right)^{T} \in Q$ are any arbitrary but fixed vectors. The feasible set of (NVP) is given by $S_{0}=\left\{x \in \mathbb{R}^{n}:-g(x)-s(x \mid D) q \in Q\right\}$.

\section{Remark 3.1.}

(i) If we take $m=1, p=m, K=\mathbf{R}_{+}, k=1, f(x)=k(x), Q=\mathbf{R}_{+}^{m}$ and $D=\{0\}$, then our Problem (NVP) reduces to the problem $\left(\mathrm{P}^{\prime}\right)$ considered by Schechter [11].

(ii) If we take $m=1, p=m, K=\mathbb{R}_{+}, k=1, Q=\mathbb{R}_{+}^{m}$ and $q=(1,1, \ldots, 1)^{T} \in Q$ then our Problem (NVP) reduces to the problem (NP) considered by Husain et al. [9] where $D_{j}=D$ for $j=1,2, \ldots, m$.

(iii) If we interchange the roles of $m$ and $p$ and take $K=\mathbb{R}_{+}^{p}, Q=\mathbb{R}_{+}^{m}, k=$ $(1,1, \ldots, 1) \in \operatorname{int} K$ and $q=(1,1, \ldots, 1) \in Q$, then our problem (NVP) reduces to (NP) considered by Husain et al. [8] where $C^{i}=C$ for each $i=$ $1,2, \ldots, p$ and $D^{j}=D$ for each $j=1,2, \ldots, m$.

Definition 3.2. A point $\bar{x} \in S_{0}$ is called

(i) a weak minimum of (NVP), if for every $x \in S_{0}$,

$$
f(\bar{x})+s(\bar{x} \mid C) k-f(x)-s(x \mid C) k \notin \operatorname{int} K .
$$

(ii) a minimum of (NVP), if for every $x \in S_{0}$,

$$
f(\bar{x})+s(\bar{x} \mid C) k-f(x)-s(x \mid C) k \notin K \backslash\{0\} .
$$

(iii) a strong minimum of (NVP), if for every $x \in S_{0}$,

$$
f(x)+s(x \mid C) k-f(\bar{x})-s(\bar{x} \mid C) k \in K .
$$

Lemma 3.3. Consider the problem

$K$-Minimize $\Phi(x)$

subject to

$$
-\Psi(x) \in Q
$$

where $\Phi: \mathbb{R}^{n} \longrightarrow \mathbb{R}^{m}$ and $\Psi: \mathbb{R}^{n} \longrightarrow \mathbb{R}^{p}$ are locally Lipschitz functions. Let $\bar{x}$ be a weak minimum of this problem, then there exists $(0,0) \neq(\lambda, \mu) \in K^{+} \times Q^{+}$such that

$$
\begin{gathered}
0 \in \partial^{c}\left(\lambda^{T} \Phi+\mu^{T} \Psi\right)(\bar{x}) \\
\left(\mu^{T} \Psi\right)(\bar{x})=0 .
\end{gathered}
$$


Proof. This Lemma can be proved by taking $F=\Phi, G=\Psi, Q=K, S=Q$ and $T=\phi$ (the empty set) in the problem (P) considered by Craven [1] and applying Theorem 2 given by him.

We now establish Fritz-John type necessary optimality conditions for the problem (NVP), based on the above lemma.

Theorem 3.4. Let $\bar{x} \in S_{0}$ be a weak minimum of (NVP). Then there exist $\bar{\lambda} \in$ $K^{+}, \bar{\mu} \in Q^{+}$with $(\bar{\lambda}, \bar{\mu}) \neq 0$ and $\bar{z} \in \partial s(\bar{x} \mid C), \bar{w} \in \partial s(\bar{x} \mid D)$ such that

$$
\begin{gathered}
\nabla\left(\bar{\lambda}^{T} f\right)(\bar{x})+\bar{z}\left(\bar{\lambda}^{T} k\right)+\nabla\left(\bar{\mu}^{T} g\right)(\bar{x})+\bar{w}\left(\bar{\mu}^{T} q\right)=0 \\
\left(\bar{\mu}^{T} g\right)(\bar{x})+\bar{x}^{T} \bar{w}\left(\bar{\mu}^{T} q\right)=0 .
\end{gathered}
$$

Proof. Let $\bar{x} \in S_{0}$ be a weak minimum of (NVP) and let $\Phi: \mathbb{R}^{n} \longrightarrow \mathbb{R}^{m}$ and $\Psi: \mathbb{R}^{n} \longrightarrow \mathbb{R}^{p}$ be such that

$$
\Phi(x)=f(x)+s(x \mid C) k \quad \text { and } \quad \Psi(x)=g(x)+s(x \mid D) q .
$$

Since $s(\cdot \mid C)$ and $s(\cdot \mid D)$ are convex functions, therefore they are locally Lipschitz at any $x \in \mathbb{R}^{n}$ and hence $s(\cdot \mid C) k$ and $s(\cdot \mid D) q$ are vector-valued locally Lipschitz functions at any $x \in \mathbb{R}^{n}$ and because $f$ and $g$ are vector-valued continuously differentiable functions, so they are vector-valued locally Lipschitz at any $x \in \mathbb{R}^{n}$. Also sum of two locally Lipschitz functions is locally Lipschitz, therefore $\Phi$ and $\Psi$ are vector-valued locally Lipschitz functions.

Now using Lemma 3.3 , there exist $\bar{\lambda} \in K^{+}, \bar{\mu} \in Q^{+}$with $(\bar{\lambda}, \bar{\mu}) \neq 0$ such that

$$
\begin{gathered}
0 \in \partial^{c}\left(\bar{\lambda}^{T} \Phi+\bar{\mu}^{T} \Psi\right)(\bar{x}) \subseteq \partial^{c}\left(\bar{\lambda}^{T} \Phi\right)(\bar{x})+\partial^{c}\left(\bar{\mu}^{T} \Psi\right)(\bar{x}) \\
\left(\bar{\mu}^{T} \Psi\right)(\bar{x})=0 .
\end{gathered}
$$

That is,

$$
\begin{gathered}
0 \in\left\{\nabla\left(\bar{\lambda}^{T} f\right)(\bar{x})\right\}+\partial^{c} s(\bar{x} \mid C)\left(\bar{\lambda}^{T} k\right)+\left\{\nabla\left(\bar{\mu}^{T} g\right)(\bar{x})\right\}+\partial^{c} s(\bar{x} \mid D)\left(\bar{\mu}^{T} q\right) \\
\left(\bar{\mu}^{T} g\right)(\bar{x})+s(\bar{x} \mid D)\left(\bar{\mu}^{T} q\right)=0 .
\end{gathered}
$$

Due to convexity of support function $\partial^{c} s(\bar{x} \mid C)=\partial s(\bar{x} \mid C)$ and $\partial^{c} s(\bar{x} \mid D)=$ $\partial s(\bar{x} \mid D)$. Thus there exist $\bar{z} \in \partial s(\bar{x} \mid C)$ and $\bar{w} \in \partial s(\bar{x} \mid D)$ such that

$$
\begin{gathered}
\nabla\left(\bar{\lambda}^{T} f\right)(\bar{x})+\bar{z}\left(\bar{\lambda}^{T} k\right)+\nabla\left(\bar{\mu}^{T} g\right)(\bar{x})+\bar{w}\left(\bar{\mu}^{T} q\right)=0 \\
\left(\bar{\mu}^{T} g\right)(\bar{x})+\bar{x}^{T} \bar{w}\left(\bar{\mu}^{T} q\right)=0 .
\end{gathered}
$$


On the lines of Suneja et al. [15], we establish the following Kuhn-Tucker type necessary optimality conditions for the problem (NVP).

Theorem 3.5. Let $\bar{x} \in S_{0}$ be a weak minimum of (NVP). Then there exist $\bar{\lambda} \in$ $K^{+}, \bar{\mu} \in Q^{+}$with $(\bar{\lambda}, \bar{\mu}) \neq 0$ and $\bar{z} \in \partial s(\bar{x} \mid C), \bar{w} \in \partial s(\bar{x} \mid D)$ such that (3.1) and (3.2) hold.

Moreover if $g$ is $Q$-convex at $\bar{x}$ and there exists $x^{*} \in \mathbb{R}^{n}$ such that

$$
\left(\bar{\mu}^{T} g\right)\left(x^{*}\right)+s\left(x^{*} \mid D\right)\left(\bar{\mu}^{T} q\right)<0
$$

Then $\bar{\lambda} \neq 0$.

Proof. Let $\bar{x}$ be a weak minimum of (NVP), then we invoke Theorem 3.4 to deduce that there exist $\bar{\lambda} \in K^{+}$and $\bar{\mu} \in Q^{+}$with $(\bar{\lambda}, \bar{\mu}) \neq 0$ and $\bar{z} \in \partial s(\bar{x} \mid C), \bar{w} \in \partial s(\bar{x} \mid D)$ such that (3.1) and (3.2) hold.

Suppose now that $g$ is $Q$-convex at $\bar{x}$ and there exists $x^{*} \in \mathbb{R}^{n}$ such that

$$
\left(\bar{\mu}^{T} g\right)\left(x^{*}\right)+s\left(x^{*} \mid D\right)\left(\bar{\mu}^{T} q\right)<0 .
$$

We have to prove that $\bar{\lambda} \neq 0$.

Let, if possible, $\bar{\lambda}=0$, then $\bar{\mu} \neq 0$ and (3.1) reduces to

$$
\nabla\left(\bar{\mu}^{T} g\right)(\bar{x})+\bar{w}\left(\bar{\mu}^{T} q\right)=0 .
$$

Now as $g$ is $Q$-convex at $\bar{x}$, hence

$$
g\left(x^{*}\right)-g(\bar{x})-\nabla g(\bar{x})\left(x^{*}-\bar{x}\right) \in Q .
$$

Since $\bar{w} \in \partial s(\bar{x} \mid D)$, therefore

$$
s\left(x^{*} \mid D\right)-s(\bar{x} \mid D) \geq\left(x^{*}-\bar{x}\right)^{T} \bar{w},
$$

which implies that

$$
\left[s\left(x^{*} \mid D\right)-s(\bar{x} \mid D)-\left(x^{*}-\bar{x}\right)^{T} \bar{w}\right] q \in Q .
$$

Adding relations (3.5) and (3.6) and using the fact that $\bar{\mu} \in Q^{+}$and $\bar{w} \in \partial s(\bar{x} \mid D)$, we get

$$
\begin{aligned}
\left(\bar{\mu}^{T} g\right)\left(x^{*}\right) & +s\left(x^{*} \mid D\right)\left(\bar{\mu}^{T} q\right)-\left(\bar{\mu}^{T} g\right)(\bar{x})-\bar{x}^{T} \bar{w}\left(\bar{\mu}^{T} q\right)-\left(x^{*}-\bar{x}\right)^{T} \\
& \times\left[\nabla\left(\bar{\mu}^{T} g\right)(\bar{x})+\bar{w}\left(\bar{\mu}^{T} q\right)\right] \geq 0
\end{aligned}
$$

Using (3.2) and (3.4) in the above inequality, we get

$$
\left(\bar{\mu}^{T} g\right)\left(x^{*}\right)+s\left(x^{*} \mid D\right)\left(\bar{\mu}^{T} q\right) \geq 0
$$

which is a contradiction to (3.3). Hence, $\bar{\lambda} \neq 0$. 
Next we give some sufficient optimality conditions for the problem (NVP) assuming the concerned functions to be cone-convex.

Theorem 3.6. Let $\bar{x} \in S_{0}$ and suppose that there exist $\bar{\lambda} \in K^{+} \backslash\{0\}, \bar{\mu} \in Q^{+}$, $\bar{z} \in \partial s(\bar{x} \mid C)$ and $\bar{w} \in \partial s(\bar{x} \mid D)$ such that (3.1) and (3.2) hold. If $f$ be $K$-convex and $g$ be $Q$-convex at $\bar{x}$, then $\bar{x}$ is a weak minimum of (NVP).

Proof. Let $x \in S_{0}$ be any arbitrary point, then

$$
-g(x)-s(x \mid D) q \in Q .
$$

Since $\bar{w} \in \partial s(\bar{x} \mid D)$, therefore

$$
\left[s(x \mid D)-s(\bar{x} \mid D)-(x-\bar{x})^{T} \bar{w}\right] q \in Q .
$$

As $g$ is $Q$-convex at $\bar{x}$, therefore

$$
g(x)-g(\bar{x})-\nabla g(\bar{x})(x-\bar{x}) \in Q .
$$

Adding (3.7), (3.8) and (3.9), we get

$$
-g(\bar{x})-s(\bar{x} \mid D) q-\nabla g(\bar{x})(x-\bar{x})-(x-\bar{x})^{T} \bar{w} q \in Q .
$$

Using $\bar{\mu} \in Q^{+}$in above relation, we get

$$
-\left(\bar{\mu}^{T} g\right)(\bar{x})-s(\bar{x} \mid D)\left(\bar{\mu}^{T} q\right)-(x-\bar{x})^{T}\left[\nabla\left(\bar{\mu}^{T} g\right)(\bar{x})+\bar{w}\left(\bar{\mu}^{T} q\right)\right] \geq 0 .
$$

Using the fact that $\bar{w} \in \partial s(\bar{x} \mid D)$ in above inequality and then applying (3.1) and (3.2) we have

$$
(x-\bar{x})^{T}\left[\nabla\left(\bar{\lambda}^{T} f\right)(\bar{x})+\bar{z}\left(\bar{\lambda}^{T} k\right)\right] \geq 0 .
$$

Since $\bar{z} \in \partial s(\bar{x} \mid C)$, therefore

$$
\left.\left[s(x \mid C)-s(\bar{x} \mid C)-(x-\bar{x})^{T} \bar{z}\right)\right] k \in K .
$$

Since $f$ is $K$-convex at $\bar{x}$, therefore

$$
f(x)-f(\bar{x})-\nabla f(\bar{x})(x-\bar{x}) \in K .
$$

Adding the above two relations and using the fact that $\bar{\lambda} \in K^{+} \backslash\{0\}$, we get

$$
\begin{aligned}
\left(\bar{\lambda}^{T} f\right)(x) & +s(x \mid C)\left(\bar{\lambda}^{T} k\right)-\left(\bar{\lambda}^{T} f\right)(\bar{x})-s(\bar{x} \mid C)\left(\bar{\lambda}^{T} k\right)-(x-\bar{x})^{T} \\
& \times\left[\nabla\left(\bar{\lambda}^{T} f\right)(\bar{x})+\bar{z}\left(\bar{\lambda}^{T} k\right)\right] \geq 0 .
\end{aligned}
$$

Adding (3.10) and (3.11), we have

$$
\left(\bar{\lambda}^{T} f\right)(\bar{x})+s(\bar{x} \mid C)\left(\bar{\lambda}^{T} k\right)-\left(\bar{\lambda}^{T} f\right)(x)-s(x \mid C)\left(\bar{\lambda}^{T} k\right) \leq 0 .
$$

Again since $\bar{\lambda} \in K^{+} \backslash\{0\}$, we have

$$
f(\bar{x})+s(\bar{x} \mid C) k-f(x)-s(x \mid C) k \notin \operatorname{int} K .
$$

Hence, $\bar{x}$ is a weak minimum for (NVP).

Now we give an example to illustrate the above theorem. 
Example 3.7. Let $f: \mathbb{R} \longrightarrow \mathbb{R}^{2}$ and $g: \mathbb{R} \longrightarrow \mathbb{R}^{2}$

$$
\begin{gathered}
f(x)=\left(\begin{array}{l}
f_{1}(x) \\
f_{2}(x)
\end{array}\right)=\left(\begin{array}{c}
1-x^{2} \\
x
\end{array}\right), C=[-1,1], \quad s(x \mid C)=\left\{\begin{array}{cc}
-x & x \leq 0 \\
x & x>0
\end{array}\right. \\
K=\left\{\left(\begin{array}{l}
x_{1} \\
x_{2}
\end{array}\right) \in \mathbb{R}^{2}: x_{1} \leq 0, x_{1} \leq-x_{2}\right\}, \quad k=\left(\begin{array}{c}
-1 \\
0
\end{array}\right) \in \operatorname{int} K . \\
g(x)=\left(\begin{array}{l}
g_{1}(x) \\
g_{2}(x)
\end{array}\right)=\left(\begin{array}{c}
2-7 x^{4} \\
x^{4}+x+2
\end{array}\right), D=[0,1], \quad s(x \mid D)= \begin{cases}0 & x \leq 0 \\
x & x>0\end{cases} \\
Q=\left\{\left(\begin{array}{l}
x_{1} \\
x_{2}
\end{array}\right) \in \mathbb{R}^{2}: x_{1} \leq x_{2} \leq-x_{1}\right\}, \quad q=\left(\begin{array}{c}
-11 / 4 \\
1
\end{array}\right) \in Q .
\end{gathered}
$$

Now, the problem (NVP) is as follows

(NVP) $\quad K$-Minimize $\left(f_{1}(x)+s(x \mid C) k_{1}, f_{2}(x)+s(x \mid C) k_{2}\right)^{T}$ subject to

where

$$
-\left(g_{1}(x)+s(x \mid D) q_{1}, g_{2}(x)+s(x \mid D) q_{2}\right)^{T} \in Q,
$$

$$
f_{1}(x)+s(x \mid C) k_{1}=\left\{\begin{array}{ll}
1+x-x^{2} & x \leq 0 \\
1-x-x^{2} & x>0
\end{array}, \quad f_{2}(x)+s(x \mid C) k_{2}=x\right.
$$

$$
\begin{aligned}
& g_{1}(x)+s(x \mid D) q_{1}=\left\{\begin{array}{ll}
2-7 x^{4} & x \leq 0 \\
2-\frac{11}{4} x-7 x^{4} & x>0
\end{array},\right. \\
& g_{2}(x)+s(x \mid D) q_{2}= \begin{cases}x^{4}+x+2 & x \leq 0 \\
x^{4}+2 x+2 & x>0\end{cases}
\end{aligned}
$$

The feasible set of the problem (NVP) is $S_{0}=\left[-\frac{1}{2}, 0\right]$. Let $\bar{x}=0$.

Then $f$ is $K$-convex at $\bar{x}$, because for every $x \in \mathbb{R}$, we have

$$
f(x)-f(\bar{x})-\nabla f(\bar{x})(x-\bar{x})=\left(\begin{array}{c}
-x^{2} \\
0
\end{array}\right) \in K
$$

And $g$ is $Q$-convex at $\bar{x}$, because for every $x \in \mathbb{R}$, we have

$$
\begin{gathered}
g(x)-g(\bar{x})-\nabla g(\bar{x})(x-\bar{x})=\left(\begin{array}{c}
-7 x^{4} \\
x^{4}
\end{array}\right) \in Q(\text { Fig. 1) } . \\
K^{+}=\left\{\left(\begin{array}{c}
x_{1} \\
x_{2}
\end{array}\right) \in \mathbb{R}^{2}: x_{1} \leq x_{2} \leq 0\right\}, Q^{+}=Q \\
\partial s(\bar{x} \mid C)=[-1,1] \text { and } \partial s(\bar{x} \mid D)=[0,1] .
\end{gathered}
$$




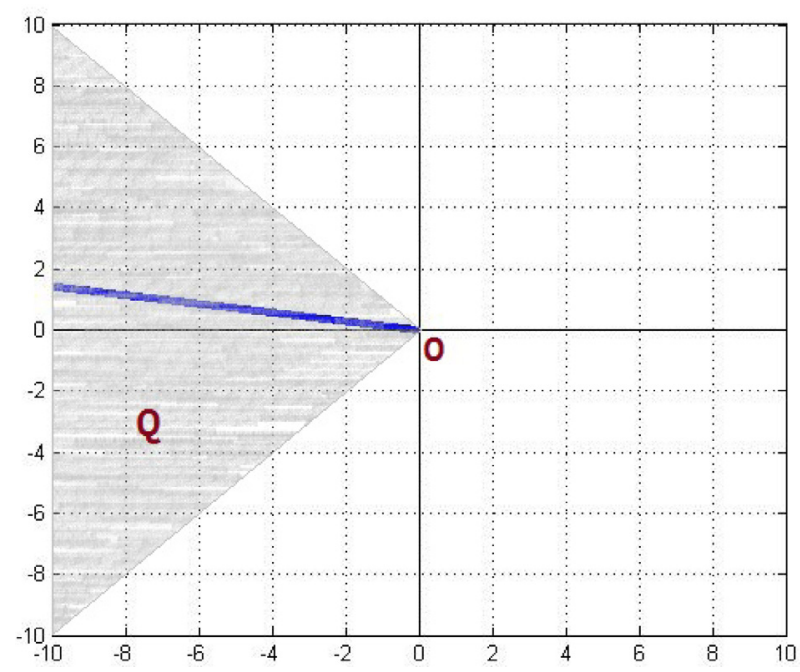

Figure 1. The line represents $g(x)-g(\bar{x})-\nabla g(\bar{x})(x-\bar{x})$ and the shaded region represents $Q$.

Also there exist $\bar{\lambda}=(-1,-1)^{T} \in K^{+} \backslash\{0\}, \bar{\mu}=(-1,1)^{T} \in Q^{+}, \bar{z}=-\frac{1}{4} \in$ $\partial s(\bar{x} \mid C), \bar{w}=\frac{1}{15} \in \partial s(\bar{x} \mid D)$ such that

$$
\begin{gathered}
\nabla\left(\bar{\lambda}^{T} f\right)(\bar{x})+\bar{z}\left(\bar{\lambda}^{T} k\right)+\nabla\left(\bar{\mu}^{T} g\right)(\bar{x})+\bar{w}\left(\bar{\mu}^{T} q\right)=0 \\
\left(\bar{\mu}^{T} g\right)(\bar{x})+\bar{x}^{T} \bar{w}\left(\bar{\mu}^{T} q\right)=0 .
\end{gathered}
$$

Therefore, $\bar{x}$ is a weak minimum of problem (NVP).

Theorem 3.8. Let $\bar{x} \in S_{0}$ and suppose that there exist $\bar{\lambda} \in K^{s+}, \bar{\mu} \in Q^{+}, \bar{z} \in$ $\partial s(\bar{x} \mid C)$ and $\bar{w} \in \partial s(\bar{x} \mid D)$ such that (3.1) and (3.2) hold. If $f$ be $K$-convex and $g$ be $Q$-convex at $\bar{x}$, then $\bar{x}$ is a minimum of $(N V P)$.

Proof. Proceeding on the lines of proof of Theorem 3.6, we have, for every $x \in S_{0}$

$$
\left(\bar{\lambda}^{T} f\right)(\bar{x})+s(\bar{x} \mid C)\left(\bar{\lambda}^{T} k\right)-\left(\bar{\lambda}^{T} f\right)(x)-s(x \mid C)\left(\bar{\lambda}^{T} k\right) \leq 0 .
$$

Since $\bar{\lambda} \in K^{s+}$, therefore we have

$$
f(\bar{x})+s(\bar{x} \mid C) k-f(x)-s(x \mid C) k \notin K \backslash\{0\} .
$$

Hence, $\bar{x}$ is a minimum for (NVP).

Theorem 3.9. Let $\bar{x} \in S_{0}$ and suppose that there exist $\bar{\mu} \in Q^{+}, \bar{z} \in \partial s(\bar{x} \mid C)$ and $\bar{w} \in \partial s(\bar{x} \mid D)$ such that (3.1) and (3.2) hold (with $\bar{\lambda}$ replaced by $\lambda$ ) for all $\lambda \in K^{+}$. If $f$ be $K$-convex and $g$ be $Q$-convex at $\bar{x} \in S_{0}$, then $\bar{x}$ is a strong minimum of $(N V P)$. 
Proof. Proceeding on the lines of proof of Theorem 3.6, we have, for every $x \in S_{0}$

$$
\left(\lambda^{T} f\right)(x)+s(x \mid C)\left(\lambda^{T} k\right)-\left(\lambda^{T} f\right)(\bar{x})-s(\bar{x} \mid C)\left(\lambda^{T} k\right) \geq 0 \quad \forall \lambda \in K^{+},
$$

which implies that

$$
f(x)+s(x \mid C) k-f(\bar{x})-s(\bar{x} \mid C) k \in\left(K^{+}\right)^{+}=K .
$$

Hence, $\bar{x}$ is a strong minimum for (NVP).

Sufficient optimality conditions are also proved under (strictly, strongly) conepseudoconvexity and cone-quasiconvexity assumptions.

Theorem 3.10. Let $\bar{x} \in S_{0}$ and suppose that there exist $\bar{\lambda} \in K^{+} \backslash\{0\}, \bar{\mu} \in Q^{+}$, $\bar{z} \in \partial s(\bar{x} \mid C)$ and $\bar{w} \in \partial s(\bar{x} \mid D)$ such that (3.1) and (3.2) hold. If $f(\cdot)+(\cdot)^{T} \bar{z} k$ is $K-$ pseudoconvex and $g(\cdot)+(\cdot)^{T} \bar{w} q$ is $Q$-quasiconvex at $\bar{x}$, then $\bar{x}$ is a weak minimum of $(N V P)$.

Proof. Let $x \in S_{0}$ be any arbitrary point, then

$$
-g(x)-s(x \mid D) q \in Q
$$

and because $\bar{w} \in D$, we have $s(x \mid D) \geq x^{T} \bar{w}$, which implies that

$$
\left[s(x \mid D)-x^{T} \bar{w}\right] q \in Q
$$

Adding (3.12) and (3.13) and using $\bar{\mu} \in Q^{+}$, we get

$$
\left(\bar{\mu}^{T} g\right)(x)+x^{T} \bar{w}\left(\bar{\mu}^{T} q\right) \leq 0 .
$$

From (3.2) and above inequality, we have

$$
\left(\bar{\mu}^{T} g\right)(x)+x^{T} \bar{w}\left(\bar{\mu}^{T} q\right)-\left(\bar{\mu}^{T} g\right)(\bar{x})-\bar{x}^{T} \bar{w}\left(\bar{\mu}^{T} q\right) \leq 0 .
$$

If $\bar{\mu} \neq 0$, then

$$
g(x)+\left(x^{T} \bar{w}\right) q-g(\bar{x})-\left(\bar{x}^{T} \bar{w}\right) q \notin \operatorname{int} Q .
$$

Since $g(\cdot)+(\cdot)^{T} \bar{w} q$ is $Q$-quasiconvex at $\bar{x}$, therefore

$$
-\nabla g(\bar{x})(x-\bar{x})-(x-\bar{x})^{T} \bar{w} q \in Q .
$$


Using $\bar{\mu} \in Q^{+}$, we have

$$
-(x-\bar{x})^{T}\left[\nabla\left(\bar{\mu}^{T} g\right)(\bar{x})+\bar{w}\left(\bar{\mu}^{T} q\right)\right] \geq 0 .
$$

If $\bar{\mu}=0$, then also the above inequality holds. Now using above inequality in (3.1), we get

$$
-(x-\bar{x})^{T}\left[\nabla\left(\bar{\lambda}^{T} f\right)(\bar{x})+\bar{z}\left(\bar{\lambda}^{T} k\right)\right] \leq 0 .
$$

Since $\bar{\lambda} \in K^{+} \backslash\{0\}$, we have

$$
-\nabla f(\bar{x})(x-\bar{x})-(x-\bar{x})^{T} \bar{z} k \notin \operatorname{int} K
$$

Since $f(\cdot)+(\cdot)^{T} \bar{z} k$ is $K$-pseudoconvex at $\bar{x}$, therefore

$$
-\left[f(x)+\left(x^{T} \bar{z}\right) k-f(\bar{x})-\left(\bar{x}^{T} \bar{z}\right) k\right] \notin \operatorname{int} K
$$

Thus, using (2.1) there exists $\lambda^{*} \in K^{+} \backslash\{0\}$, such that

$$
-\left(\lambda^{* T} f\right)(x)-x^{T} \bar{z}\left(\lambda^{* T} k\right)+\left(\lambda^{* T} f\right)(\bar{x})+\bar{x}^{T} \bar{z}\left(\lambda^{* T} k\right) \leq 0
$$

Since $\bar{z} \in \partial s(\bar{x} \mid C)$, we have

$$
\left[s(x \mid C)-s(\bar{x} \mid C)-x^{T} \bar{z}+\bar{x}^{T} \bar{z}\right] k \in K .
$$

Using $\lambda^{*} \in K^{+} \backslash\{0\}$, we get

$$
\left[-s(x \mid C)+s(\bar{x} \mid C)+x^{T} \bar{z}-\bar{x}^{T} \bar{z}\right]\left(\lambda^{* T} k\right) \leq 0
$$

Adding (3.16) and (3.17), we get

$$
\left(\lambda^{* T} f\right)(\bar{x})+s(\bar{x} \mid C)\left(\lambda^{* T} k\right)-\left(\lambda^{* T} f\right)(x)-s(x \mid C)\left(\lambda^{* T} k\right) \leq 0,
$$

which implies that

$$
f(\bar{x})+s(\bar{x} \mid C) k-f(x)-s(x \mid C) k \notin \operatorname{int} K .
$$

Hence, $\bar{x}$ is a weak minimum for (NVP).

Below we give an example to illustrate the above theorem. 
Example 3.11. Let $f: \mathbb{R} \longrightarrow \mathbb{R}^{2}$ and $g: \mathbb{R} \longrightarrow \mathbb{R}^{2}$

$$
\begin{gathered}
f(x)=\left(\begin{array}{l}
f_{1}(x) \\
f_{2}(x)
\end{array}\right)=\left(\begin{array}{c}
x \\
x e^{x}
\end{array}\right), C=[-1,1], \quad s(x \mid C)=\left\{\begin{array}{cc}
-x & x \leq 0 \\
x & x>0
\end{array}\right. \\
K=\left\{\left(\begin{array}{l}
x_{1} \\
x_{2}
\end{array}\right) \in \mathbb{R}^{2}:-x_{2} \leq x_{1} \leq x_{2}\right\}, \quad k=\left(\begin{array}{c}
1 / 2 \\
1
\end{array}\right) \in \operatorname{int} K . \\
g(x)=\left(\begin{array}{l}
g_{1}(x) \\
g_{2}(x)
\end{array}\right)=\left(\begin{array}{c}
x \\
(x+1)^{3}
\end{array}\right), D=[1,2], \quad s(x \mid D)= \begin{cases}x & x \leq 0 \\
2 x & x>0\end{cases} \\
Q=\left\{\left(\begin{array}{l}
x_{1} \\
x_{2}
\end{array}\right) \in \mathbb{R}^{2}: x_{1} \leq 0, x_{1} \leq-x_{2}\right\}, \quad q=\left(\begin{array}{c}
-1 / 4 \\
-1 / 8
\end{array}\right) \in Q .
\end{gathered}
$$

Now, the problem (NVP) is as follows

$(\mathrm{NVP}) \quad K$-Minimize $\left(f_{1}(x)+s(x \mid C) k_{1}, f_{2}(x)+s(x \mid C) k_{2}\right)^{T}$ subject to

where

$$
-\left(g_{1}(x)+s(x \mid D) q_{1}, g_{2}(x)+s(x \mid D) q_{2}\right)^{T} \in Q,
$$

$f_{1}(x)+s(x \mid C) k_{1}=\left\{\begin{array}{ll}x / 2 & x \leq 0 \\ 3 x / 2 & x>0\end{array}, \quad f_{2}(x)+s(x \mid C) k_{2}= \begin{cases}x\left(e^{x}-1\right) & x \leq 0 \\ x\left(e^{x}+1\right) & x>0\end{cases}\right.$

$$
g_{1}(x)+s(x \mid D) q_{1}=3 x / 4, \quad g_{2}(x)+s(x \mid D) q_{2}=(x+1)^{3}-x / 4 .
$$

The feasible set of the problem (NVP) is $S_{0}=\left[-\frac{1}{2}, 0\right]$. Let $\bar{x}=0$.

$$
\begin{gathered}
K^{+}=K, Q^{+}=\left\{\left(\begin{array}{l}
x_{1} \\
x_{2}
\end{array}\right) \in \mathbb{R}^{2}: x_{1} \leq x_{2} \leq 0\right\}, \\
\partial s(\bar{x} \mid C)=[-1,1] \text { and } \partial s(\bar{x} \mid D)=[1,2] .
\end{gathered}
$$

Also $f(\cdot)+(\cdot)^{T} \bar{z} k$ is $K$-pseudoconvex at $\bar{x}$, because for every $x \in \mathbb{R}$, we have

$\operatorname{int} K$ (Fig. 2b)

$$
\begin{aligned}
& -\nabla f(\bar{x})(x-\bar{x})-(x-\bar{x})^{T} \bar{z} k=\left(\begin{array}{c}
-5 x / 4 \\
-3 x / 2
\end{array}\right) \notin \operatorname{int} \text { (Fig. 2a) } \\
\Longrightarrow & x \geq 0 \\
\Longrightarrow & -\left[f(x)+\left(x^{T} \bar{z}\right) k-f(\bar{x})-\left(\bar{x}^{T} \bar{z}\right) k\right]=\left(\begin{array}{c}
-5 x / 4 \\
-x\left(e^{x}+1 / 2\right)
\end{array}\right) \notin
\end{aligned}
$$

And $g(\cdot)+(\cdot)^{T} \bar{w} q$ is $Q$-quasiconvex at $\bar{x}$, because for every $x \in \mathbb{R}$, we have

$\operatorname{int} Q$ (Fig. 3a)

$$
g(x)+\left(x^{T} \bar{w}\right) q-g(\bar{x})-\left(\bar{x}^{T} \bar{w}\right) q=\left(\begin{array}{c}
3 x / 4 \\
(x+1)^{3}-x / 8-1
\end{array}\right) \notin
$$

$$
\begin{aligned}
& \Longrightarrow x \geq 0 \\
& \Longrightarrow-\nabla g(\bar{x})(x-\bar{x})-(x-\bar{x})^{T} \bar{w} q=\left(\begin{array}{c}
-3 x / 4 \\
-23 x / 8
\end{array}\right) \in Q \text { (Fig. 3b) . }
\end{aligned}
$$




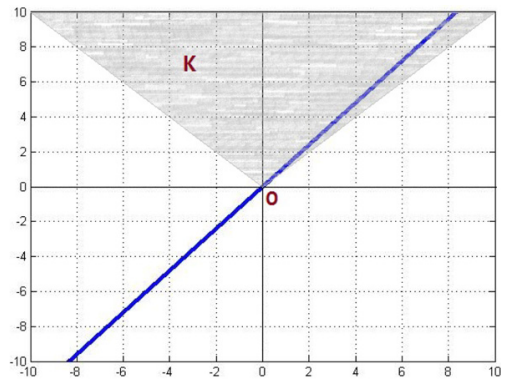

(a) The line represents $-\nabla f(\bar{x})(x-$ $\bar{x})-(x-\bar{x})^{T} \bar{z} k$ and the shaded region represents $K$.

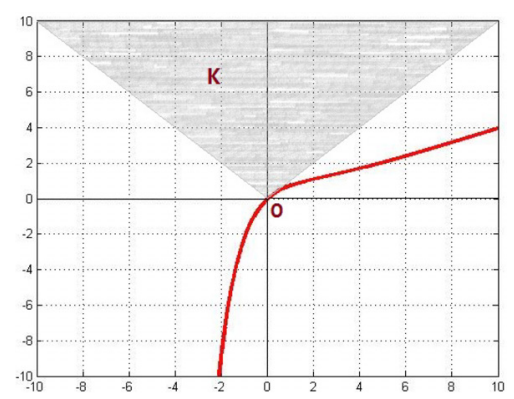

(b) The curve represents $[-f(x)+$ $\left(x^{T} \bar{z}\right) k-f(\bar{x})-\left(\bar{x}^{T} \bar{z}\right) k$ and the shaded region represents $K$.

Figure 2.

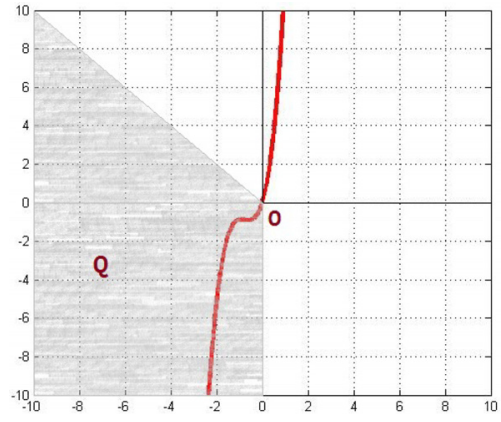

(a) The curve represents $g(x)+$ $\left(x^{T} \bar{w}\right) q-g(\bar{x})-\left(\bar{x}^{T} \bar{w}\right) q$ and the shaded region represents $Q$.

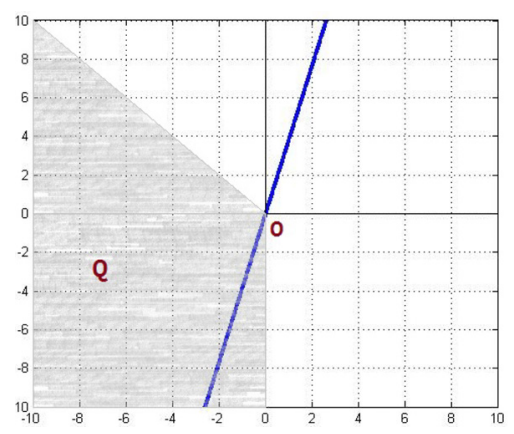

(b) The line represents $-\nabla g(\bar{x})(x-$ $\bar{x})-(x-\bar{x})^{T} \bar{w} q$ and the shaded region represents $Q$.

Figure 3.

There exist $\bar{\lambda}=\left(0, \frac{1}{2}\right)^{T} \in K^{+} \backslash\{0\}, \bar{\mu}=(-1,0)^{T} \in Q^{+}, \bar{z}=\frac{1}{2} \in$ $\partial s(\bar{x} \mid C), \bar{w}=1 \in \partial s(\bar{x} \mid D)$ such that

$$
\begin{gathered}
\nabla\left(\bar{\lambda}^{T} f\right)(\bar{x})+\bar{z}\left(\bar{\lambda}^{T} k\right)+\nabla\left(\bar{\mu}^{T} g\right)(\bar{x})+\bar{w}\left(\bar{\mu}^{T} q\right)=0 \\
\left(\bar{\mu}^{T} g\right)(\bar{x})+\bar{x}^{T} \bar{w}\left(\bar{\mu}^{T} q\right)=0 .
\end{gathered}
$$

Therefore, $\bar{x}$ is a weak minimum of problem (NVP).

Theorem 3.12. Let $\bar{x} \in S_{0}$ and suppose that there exist $\bar{\lambda} \in K^{s+}, \bar{\mu} \in Q^{+}$, $\bar{z} \in \partial s(\bar{x} \mid C)$ and $\bar{w} \in \partial s(\bar{x} \mid D)$ such that (3.1) and (3.2) hold. If $f(\cdot)+(\cdot)^{T} \bar{z} k$ is strictly $K$-pseudoconvex and $g(\cdot)+(\cdot)^{T} \bar{w} q$ is $Q$-quasiconvex at $\bar{x}$, then $\bar{x}$ is a minimum of $(N V P)$. 
Proof. Proceeding on the lines of proof of Theorem 3.10 and using the fact that $f(\cdot)+(\cdot)^{T} \bar{z} k$ is strictly $K$-pseudoconvex at $\bar{x}$, we get that, for every $x \in S_{0}$

$$
-\left[f(x)+\left(x^{T} \bar{z}\right) k-f(\bar{x})-\left(\bar{x}^{T} \bar{z}\right) k\right] \notin K \backslash\{0\} .
$$

So, there exists $\lambda^{*} \in K^{s+}$, such that

$$
-\left(\lambda^{* T} f\right)(x)-x^{T} \bar{z}\left(\lambda^{* T} k\right)+\left(\lambda^{* T} f\right)(\bar{x})+\bar{x}^{T} \bar{z}\left(\lambda^{* T} k\right) \leq 0
$$

Since $\bar{z} \in \partial s(\bar{x} \mid C)$, we have $\left[s(x \mid C)-s(\bar{x} \mid C)-x^{T} \bar{z}+\bar{x}^{T} \bar{z}\right] k \in K$ and using that $\lambda^{*} \in K^{s+}$, we get

$$
\left[-s(x \mid C)+s(\bar{x} \mid C)+x^{T} \bar{z}-\bar{x}^{T} \bar{z}\right]\left(\lambda^{* T} k\right) \leq 0
$$

Adding the above two inequalities, we get

$$
\left(\lambda^{* T} f\right)(\bar{x})+s(\bar{x} \mid C)\left(\lambda^{* T} k\right)-\left(\lambda^{* T} f\right)(x)-s(x \mid C)\left(\lambda^{* T} k\right) \leq 0,
$$

which implies that

$$
f(\bar{x})+s(\bar{x} \mid C) k-f(x)-s(x \mid C) k \notin K \backslash\{0\} .
$$

Hence, $\bar{x}$ is a minimum for (NVP).

Theorem 3.13. Let $\bar{x} \in S_{0}$ and suppose that there exist $\bar{\lambda} \in K^{+} \backslash\{0\}, \bar{\mu} \in Q^{+}$, $\bar{z} \in \partial s(\bar{x} \mid C)$ and $\bar{w} \in \partial s(\bar{x} \mid D)$ such that (3.1) and (3.2) hold. If $f(\cdot)+(\cdot)^{T} \bar{z} k$ is strongly K-pseudoconvex and $g(\cdot)+(\cdot)^{T} \bar{w} q$ is $Q$-quasiconvex at $\bar{x}$, then $\bar{x}$ is a strong minimum of (NVP).

Proof. Proceeding on the lines of proof of Theorem 3.10 and using the fact that $f(\cdot)+(\cdot)^{T} \bar{z} k$ is strongly $K$-pseudoconvex at $\bar{x}$, we get that, for every $x \in S_{0}$

$$
f(x)+\left(x^{T} \bar{z}\right) k-f(\bar{x})-\left(\bar{x}^{T} \bar{z}\right) k \in K .
$$

Since $\bar{z} \in \partial s(\bar{x} \mid C)$, we have

$$
\left[s(x \mid C)-s(\bar{x} \mid C)-x^{T} \bar{z}+\bar{x}^{T} \bar{z}\right] k \in K .
$$

Adding the above two inequalities, we get

$$
f(x)+s(x \mid C) k-f(\bar{x})-s(\bar{x} \mid C) k \in K .
$$

Hence, $\bar{x}$ is a strong minimum for (NVP). 


\section{UNIFIED DUALITY}

The Unified Duality approach studied in this section has been proposed in the literature in order to manage both the Mond-Weir and the Wolfe type duals in a unifying framework. On the lines of Cambini and Carosi [14], we propose the following unified dual for our problem (NVP) by considering a $0-1$ parameter $\delta \in\{0,1\}$.

$$
\begin{aligned}
& \text { K-Maximize } f(u)+\left(u^{T} z\right) k+\delta\left[\left(\mu^{T} g\right)(u)+u^{T} w\left(\mu^{T} q\right)\right] k \\
& \text { subject to } \\
& \quad \nabla\left(\lambda^{T} f\right)(u)+z\left(\lambda^{T} k\right)+\nabla\left(\mu^{T} g\right)(u)+w\left(\mu^{T} q\right)=0 \\
& \quad(1-\delta)\left[\left(\mu^{T} g\right)(u)+u^{T} w\left(\mu^{T} q\right)\right] \geq 0 \\
& \quad \delta\left(\lambda^{T} k\right)=\delta, \\
& \text { where } u \in \mathbb{R}^{n}, z \in C, w \in D, \lambda \in K^{+} \backslash\{0\}, \mu \in Q^{+} .
\end{aligned}
$$

It is worth noticing here that if $\delta=0$, then the above dual becomes Mond-Weir type dual and if $\delta=1$, then it becomes Wolfe type dual.

Remark 4.1. The presence of $\delta$ in the third constraint $\delta\left(\lambda^{T} k\right)=\delta$ of our unified dual is to remark the fact that we do not need this constraint in the case of Mond-Weir dual, i.e., when $\delta=0$.

\section{Remark 4.2.}

(i) If we take $m=1, p=m, K=\mathbb{R}_{+}, Q=\mathbb{R}_{+}^{m}, z=w$, for each $j=1,2, \ldots, m$, $w^{j}=0, \mu=y$ and $\delta=1$ then our dual (UD) reduces to the dual (D") considered by Schechter [11].

(ii) Let $m=1, p=m, K=\mathbb{R}_{+}, Q=\mathbb{R}_{+}^{m}$ and $k=1$. Now if $\delta=1$ then our dual (UD) reduces to the dual (WD) and if $\delta=0$ then it reduces to the dual (M-WD), both considered by Husain et al. [9].

Now we will establish Weak Duality relations for (NVP) and its unified dual (UD).

Theorem 4.3 Weak Duality. Let $x$ be feasible for $(N V P)$ and $(u, z, w, \lambda, \mu)$ be feasible for (UD). If $f$ is $K$-convex and $g$ is $Q$-convex at $u$, then we have

$$
f(u)+\left(u^{T} z\right) k+\delta\left[\left(\mu^{T} g\right)(u)+u^{T} w\left(\mu^{T} q\right)\right] k-f(x)-s(x \mid C) k \notin \text { int } K .
$$

Proof. Let, if possible,

$$
f(u)+\left(u^{T} z\right) k+\delta\left[\left(\mu^{T} g\right)(u)+u^{T} w\left(\mu^{T} q\right)\right] k-f(x)-s(x \mid C) k \in \operatorname{int} K .
$$

Since $z \in C$, therefore $s(x \mid C) \geq x^{T} z$ and

$$
\left[s(x \mid C)-x^{T} z\right] k \in K .
$$


Adding the above two relations, we get

$$
f(u)+\left(u^{T} z\right) k+\delta\left[\left(\mu^{T} g\right)(u)+u^{T} w\left(\mu^{T} q\right)\right] k-f(x)-\left(x^{T} z\right) k \in \operatorname{int} K .
$$

Since $f$ is $K$-convex at $u$, therefore

$$
f(x)-f(u)-\nabla f(u)(x-u) \in K .
$$

Again adding the above two relations and using the fact that $\lambda \in K^{+} \backslash\{0\}$, we get

$$
-(x-u)^{T}\left[\nabla\left(\lambda^{T} f\right)(u)+z\left(\lambda^{T} k\right)\right]+\delta\left[\left(\mu^{T} g\right)(u)+u^{T} w\left(\mu^{T} q\right)\right]\left(\lambda^{T} k\right)>0 .
$$

Using the feasibility of $(u, z, w, \lambda, \mu)$ for (UD) in the above inequality, we get

$$
(x-u)^{T}\left[\nabla\left(\mu^{T} g\right)(u)+w\left(\mu^{T} q\right)\right]+\left[\left(\mu^{T} g\right)(u)+u^{T} w\left(\mu^{T} q\right)\right]>0,
$$

which implies that

$$
(x-u)^{T} \nabla\left(\mu^{T} g\right)(u)+x^{T} w\left(\mu^{T} q\right)+\left(\mu^{T} g\right)(u)>0 .
$$

Since $g$ is $Q$-convex at $u$ and $\mu \in Q^{+}$, therefore

$$
\left(\mu^{T} g\right)(x)-\left(\mu^{T} g\right)(u)-(x-u)^{T} \nabla\left(\mu^{T} g\right)(u) \geq 0 .
$$

Adding the above two inequalities, we get

$$
\left(\mu^{T} g\right)(x)+x^{T} w\left(\mu^{T} q\right)>0 .
$$

Since $x$ is feasible for (NVP), $w \in D$ and $\mu \in Q^{+}$, therefore we have

$$
\left(\mu^{T} g\right)(x)+x^{T} w\left(\mu^{T} q\right) \leq 0,
$$

which is a contradiction to (4.1) and hence the result.

Now we illustrate the above result with an example.

Example 4.4. Consider the problem in Example 3.7. The Unified dual of this problem is given by

$K$-Maximize

$$
\left(1-u^{2}-u z-\delta\left\{\left(2-7 u^{4}-\frac{11}{4} u w\right) \mu_{1}+\left(u^{4}+u+2+u w\right) \mu_{2}\right\}, u\right)^{T}
$$

subject to

$$
\begin{aligned}
& -(2 u+z) \lambda_{1}+\lambda_{2}-\left(28 u^{3}+\frac{11}{4} w\right) \mu_{1}+\left(4 u^{3}+1+w\right) \mu_{2}=0 \\
& (1-\delta)\left\{\left(2-7 u^{4}-\frac{11}{4} u w\right) \mu_{1}+\left(u^{4}+u+2+u w\right) \mu_{2}\right\} \geq 0
\end{aligned}
$$

$\delta\left(\lambda^{T} k\right)=\delta$. 
where $u \in \mathbb{R}, z \in C, w \in D, \lambda=\left(\lambda_{1}, \lambda_{2}\right)^{T} \in K^{+} \backslash\{0\}, \mu=\left(\mu_{1}, \mu_{2}\right)^{T} \in Q^{+}$.

Take $x=-\frac{1}{4}$ feasible for primal problem (NVP) and $(u, z, w, \lambda, \mu)=$ $\left(0,-\frac{1}{4}, \frac{1}{15},(-1,-1)^{T},(-1,1)^{T}\right)$ feasible for Unified dual (UD). As shown in Example 3.7, the function $f$ is $K$-convex and $g$ is $Q$-convex at $u=0$;

Here,

$f(u)+\left(u^{T} z\right) k+\delta\left[\left(\mu^{T} g\right)(u)+u^{T} w\left(\mu^{T} q\right)\right] k-f(x)-s(x \mid C) k=\left(\begin{array}{c}5 / 16 \\ 1 / 4\end{array}\right) \notin \operatorname{int} K$.

Thus, Weak Duality result (Thm. 4.3) holds.

Below we define a function which will be used to prove the next Weak Duality result.

Definition 4.5. Given the Problem (NVP) and given a vector $k \in \operatorname{int} K$, we define the following function:

$$
\begin{gathered}
\mathcal{L}: \mathbb{R}^{n} \times C \times D \times Q^{+} \longrightarrow \mathbb{R}^{m} \text { such that } \\
\mathcal{L}(x, z, w, \mu)=f(x)+\left(x^{T} z\right) k+\left[\left(\mu^{T} g\right)(x)+x^{T} w\left(\mu^{T} q\right)\right] k .
\end{gathered}
$$

Theorem 4.6 Weak Duality. Let $x$ be feasible for $(N V P)$ and $(u, z, w, \lambda, \mu)$ be feasible for (UD). Suppose the following conditions hold:

(a) If $\delta=1, \mathcal{L}(\cdot, z, w, \mu)$ is $K$-pseudoconvex at $u$, and

(b) If $\delta=0, f(\cdot)+(\cdot)^{T} z k$ is $K$-pseudoconvex and $g(\cdot)+(\cdot)^{T} w q$ is Q-quasiconvex at $u$

Then, we have

$$
f(u)+\left(u^{T} z\right) k+\delta\left[\left(\mu^{T} g\right)(u)+u^{T} w\left(\mu^{T} q\right)\right] k-f(x)-s(x \mid C) k \notin \text { intK. }
$$

Proof. Case (a). Let $\delta=1$ and let, if possible,

$$
f(u)+\left(u^{T} z\right) k+\left[\left(\mu^{T} g\right)(u)+u^{T} w\left(\mu^{T} q\right)\right] k-f(x)-s(x \mid C) k \in \operatorname{int} K .
$$

Since $z \in C$, therefore $s(x \mid C) \geq x^{T} z$ and

$$
\left[s(x \mid C)-x^{T} z\right] k \in K .
$$

Adding the above two relations, we get

$$
f(u)+\left(u^{T} z\right) k+\left[\left(\mu^{T} g\right)(u)+u^{T} w\left(\mu^{T} q\right)\right] k-f(x)-\left(x^{T} z\right) k \in \operatorname{int} K .
$$

Since $x$ is feasible for (NVP), $w \in D$ and $\mu \in Q^{+}$, therefore we have

$$
-\left[\left(\mu^{T} g\right)(x)+x^{T} w\left(\mu^{T} q\right)\right] \geq 0,
$$


and using the fact that $k \in \operatorname{int} K$ and (4.4), we get

$$
-\left[\left(\mu^{T} g\right)(x)+x^{T} w\left(\mu^{T} q\right)\right] k \in K .
$$

Adding (4.3) and (4.5), we get

$$
\begin{array}{r}
f(u)+\left(u^{T} z\right) k+\left[\left(\mu^{T} g\right)(u)+u^{T} w\left(\mu^{T} q\right)\right] k-f(x)-\left(x^{T} z\right) k \\
-\left[\left(\mu^{T} g\right)(x)+x^{T} w\left(\mu^{T} q\right)\right] k \in \operatorname{int} K .
\end{array}
$$

That is,

$$
-[\mathcal{L}(x, z, w, \mu)-\mathcal{L}(u, z, w, \mu)] \in \operatorname{int} K .
$$

Using the fact that $\mathcal{L}(\cdot, z, w, \mu)$ is $K$-pseudoconvex at $\mathrm{u}$, we get

$$
-\nabla \mathcal{L}(u, z, w, \mu)(x-u) \in \operatorname{int} K
$$

Since $\lambda \in K^{+} \backslash\{0\}$, therefore

$$
(x-u)^{T} \nabla\left(\lambda^{T} \mathcal{L}\right)(u, z, w, \mu)<0,
$$

which on using the condition $\lambda^{T} k=1$, gives

$$
(x-u)^{T}\left[\nabla\left(\lambda^{T} f\right)(u)+z\left(\lambda^{T} k\right)+\nabla\left(\mu^{T} g\right)(u)+w\left(\mu^{T} q\right)\right]<0,
$$

which is a contradiction to the fact that $(u, z, w, \lambda, \mu)$ is feasible for (UD). Hence the result.

Case (b). Let $\delta=0$, then we have to prove that,

$$
f(u)+\left(u^{T} z\right) k-f(x)-s(x \mid C) k \notin \operatorname{int} K .
$$

Since $x$ is feasible for (NVP), therefore

$$
-g(x)-s(x \mid D) q \in Q
$$

and because $w \in D$, we have

$$
\left[s(x \mid D)-x^{T} w\right] q \in Q .
$$

Adding the two and using $\mu \in Q^{+}$, we get

$$
\left(\mu^{T} g\right)(x)+x^{T} w\left(\mu^{T} q\right) \leq 0 .
$$

From the above inequality and the fact that $(u, z, w, \lambda, \mu)$ is feasible for (UD) with $\delta=0$, we have

$$
\left(\mu^{T} g\right)(x)+x^{T} w\left(\mu^{T} q\right)-\left(\mu^{T} g\right)(u)-u^{T} w\left(\mu^{T} q\right) \leq 0 .
$$

If $\mu \neq 0$, then

$$
g(x)+\left(x^{T} w\right) q-g(u)-\left(u^{T} w\right) q \notin \operatorname{int} Q .
$$


Since $g(\cdot)+(\cdot)^{T} w q$ is $Q$-quasiconvex at $u$, therefore

$$
-\nabla g(u)(x-u)-(x-u)^{T} w q \in Q .
$$

Using $\mu \in Q^{+}$, we get

$$
-(x-u)^{T}\left[\nabla\left(\mu^{T} g\right)(u)+w\left(\mu^{T} q\right)\right] \geq 0 .
$$

If $\bar{\mu}=0$, then also the above inequality holds. Now using the fact that $(u, z, w, \lambda, \mu)$ is feasible for (UD) and above inequality, we get

$$
-(x-u)^{T}\left[\nabla\left(\lambda^{T} f\right)(u)+z\left(\lambda^{T} k\right)\right] \leq 0 .
$$

Since $\lambda \in K^{+} \backslash\{0\}$, we have

$$
-\nabla f(u)(x-u)-(x-u)^{T} z k \notin \operatorname{int} K .
$$

Since $f(\cdot)+(\cdot)^{T} z k$ is $K$-pseudoconvex at $u$, therefore

$$
-\left[f(x)+\left(x^{T} z\right) k-f(u)-\left(u^{T} z\right) k\right] \notin \operatorname{int} K .
$$

Thus, using (2.1) there exists $\lambda^{*} \in K^{+} \backslash\{0\}$, such that

$$
-\left(\lambda^{* T} f\right)(x)-x^{T} z\left(\lambda^{* T} k\right)+\left(\lambda^{* T} f\right)(u)+u^{T} z\left(\lambda^{* T} k\right) \leq 0 .
$$

Since $z \in C$, we have $\left[s(x \mid C)-x^{T} z\right] k \in K$ and using that $\lambda^{*} \in$ $K^{+} \backslash\{0\}$, we get

$$
\left[-s(x \mid C)+x^{T} z\right]\left(\lambda^{* T} k\right) \leq 0 .
$$

Adding the above two inequalities, we get

$$
\left(\lambda^{* T} f\right)(u)+u^{T} z\left(\lambda^{* T} k\right)-\left(\lambda^{* T} f\right)(x)-s(x \mid C)\left(\lambda^{* T} k\right) \leq 0,
$$

which implies that

$$
f(u)+\left(u^{T} z\right) k-f(x)-s(x \mid C) k \notin \operatorname{int} K .
$$

Hence the result.

We now give the Strong Duality result.

Theorem 4.7 Strong Duality. Let $\bar{x} \in S_{0}$ be a weak minimum of (NVP). Then there exist $\bar{\lambda} \in K^{+}, \bar{\mu} \in Q^{+}$with $(\bar{\lambda}, \bar{\mu}) \neq 0$ and $\bar{z} \in \partial s(\bar{x} \mid C), \bar{w} \in \partial s(\bar{x} \mid D)$ such that (3.1) and (3.2) hold. Moreover if $g$ is $Q$-convex at $\bar{x}$ and we can find $x^{*} \in \mathbb{R}^{n}$ such that $\left(\bar{\mu}^{T} g\right)\left(x^{*}\right)+s\left(x^{*} \mid D\right)\left(\bar{\mu}^{T} q\right)<0$ then there exist $\bar{\lambda} \in K^{+} \backslash\{0\}$ and $\bar{\mu} \in Q^{+}$such that $(\bar{x}, \bar{z}, \bar{w}, \bar{\lambda}, \bar{\mu})$ is feasible for (UD). Suppose conditions of Weak Duality Theorem 4.3 or 4.6 are satisfied for each feasible solution $(u, z, w, \lambda, \mu)$ of (UD), then $(\bar{x}, \bar{z}, \bar{w}, \bar{\lambda}, \bar{\mu})$ is weak maximum for (UD). 
Proof. Since $\bar{x}$ is a weak minimum of (NVP), therefore by Theorem 3.4 there exist $\lambda^{*} \in K^{+}, \mu^{*} \in Q^{+}$with $\left(\lambda^{*}, \mu^{*}\right) \neq 0$ and $\bar{z} \in \partial s(\bar{x} \mid C)$ and $\bar{w} \in \partial s(\bar{x} \mid D)$ such that (3.1) and (3.2) hold.

Since $g$ is $Q$-convex at $\bar{x}$ and there exists $x^{*} \in \mathbb{R}^{n}$ such that $\left(\mu^{* T} g\right)\left(x^{*}\right)+$ $s\left(x^{*} \mid D\right)\left(\mu^{* T} q\right)<0$, so by applying Theorem 3.5 we can prove that $\lambda^{*} \neq 0$.

Due to the fact that $\lambda^{*} \in K^{+} \backslash\{0\}$ and $k \in \operatorname{int} K$, we have $\lambda^{* T} k>0$ and $\bar{\lambda}=\frac{1}{\lambda^{* T} k} \lambda^{*} \in K^{+} \backslash\{0\}$ and $\bar{\mu}=\frac{1}{\lambda^{* T} k} \mu^{*} \in Q^{+}$. Thus there exist $\bar{\lambda} \in K^{+} \backslash\{0\}$, $\bar{\mu} \in Q^{+}, \bar{z} \in \partial s(\bar{x} \mid C)$ and $\bar{w} \in \partial s(\bar{x} \mid D)$ such that $(\bar{x}, \bar{z}, \bar{w}, \bar{\lambda}, \bar{\mu})$ is feasible for (UD).

Now suppose the conditions of Weak Duality Theorem 4.3 or 4.6 hold for all feasible points of (UD) and let if possible $(\bar{x}, \bar{z}, \bar{w}, \bar{\lambda}, \bar{\mu})$ be not a weak maximum of (UD), then there exists a feasible point $(u, z, w, \lambda, \mu)$ of (UD) such that

$$
\begin{aligned}
f(u)+\left(u^{T} z\right) k+\delta\left[\left(\mu^{T} g\right)(u)+u^{T} w\left(\mu^{T} q\right)\right] & k-f(\bar{x})+\left(\bar{x}^{T} \bar{z}\right) k \\
& +\delta\left[\left(\bar{\mu}^{T} g\right)(\bar{x})+\bar{x}^{T} \bar{w}\left(\bar{\mu}^{T} q\right)\right] k \in \operatorname{int} K
\end{aligned}
$$

Using $\bar{\mu}=\frac{1}{\lambda^{*}{ }^{T}} \mu^{*}$, equation (3.2) and the fact that $\bar{z} \in \partial s(\bar{x} \mid C)$ in the above relation we have

$$
f(u)+\left(u^{T} z\right) k+\delta\left[\left(\mu^{T} g\right)(u)+u^{T} w\left(\mu^{T} q\right)\right] k-f(\bar{x})-s(\bar{x} \mid C) k \in \operatorname{int} K,
$$

which is a contradiction to the Weak Duality Result. Hence, $(\bar{x}, \bar{z}, \bar{w}, \bar{\lambda}, \bar{\mu})$ is a weak maximum of (UD).

In order to prove the Converse Duality result, we now obtain Fritz-John type necessary optimality conditions for a point to be a weak minimum of the following program:

(VPE) $\quad K_{1}$-Minimize $F(x)$

subject to

$$
\begin{aligned}
& -G(x) \in K_{2} \\
& H(x)=0,
\end{aligned}
$$

where $F: S \longrightarrow \mathbb{R}^{m}, G: S \longrightarrow \mathbb{R}^{p}$ and $H: S \longrightarrow \mathbb{R}^{l}$ are differentiable vectorvalued functions, $S$ is a nonempty convex subset of $\mathbb{R}^{n}, K_{1}$ and $K_{2}$ are convex cones in $\mathbb{R}^{m}$ and $\mathbb{R}^{p}$ respectively with vertices at respective origins such that int $K_{1} \neq \phi$.

Lemma 4.8. Let $x^{*}$ be a weak minimum of (VPE), then there exist $\alpha^{*} \in K_{1}^{+}$, $\nu^{*} \in K_{2}^{+}$and $\beta^{*} \in \mathbb{R}^{l}$ with $\left(\alpha^{*}, \nu^{*}, \beta^{*}\right) \neq 0$ such that

$$
\begin{gathered}
\left(x-x^{*}\right)^{T}\left[\nabla\left(\alpha^{* T} F\right)\left(x^{*}\right)+\nabla\left(\nu^{* T} G\right)\left(x^{*}\right)+\nabla\left(\beta^{* T} H\right)\left(x^{*}\right)\right] \geq 0 \text { for all } x \in S \\
\left(\nu^{* T} G\right)\left(x^{*}\right)=0 \quad \text { and }\left(\beta^{* T} H\right)\left(x^{*}\right)=0 .
\end{gathered}
$$


Proof. Let $K_{3}=\{0\} \subset \mathbb{R}^{l}$, then the problem (VPE) can be written as

$K_{1}$-Minimize $F(x)$

subject to

$$
\begin{aligned}
& -G(x) \in K_{2} \\
& -H(x) \in K_{3} .
\end{aligned}
$$

We claim that there is no $x \in S$ such that

$$
\begin{gathered}
\nabla F\left(x^{*}\right)\left(x-x^{*}\right) \in-\operatorname{int} K_{1}, \\
\nabla G\left(x^{*}\right)\left(x-x^{*}\right)+G\left(x^{*}\right) \in-K_{2}, \\
\nabla H\left(x^{*}\right)\left(x-x^{*}\right) \in-K_{3} .
\end{gathered}
$$

Let, if possible, there be an $x \in S$ which is a solution to the above system.

Now since $\mathrm{S}$ is convex, therefore for any $t \in(0,1), x^{*}+t\left(x-x^{*}\right) \in S$. Further we have

$$
\begin{aligned}
G\left(x^{*}+t\left(x-x^{*}\right)\right) & =G\left(x^{*}\right)+t \nabla G\left(x^{*}\right)\left(x-x^{*}\right)+o(t) \\
& =t\left[G\left(x^{*}\right)+\nabla G\left(x^{*}\right)\left(x-x^{*}\right)\right]+(1-t) G\left(x^{*}\right)+o(t) \in-K_{2}
\end{aligned}
$$

where $\lim _{t \downarrow 0} o(t)=0$.

Since $H\left(x^{*}\right)=0$, we get

$$
\begin{aligned}
H\left(x^{*}+t\left(x-x^{*}\right)\right) & =H\left(x^{*}\right)+t \nabla H\left(x^{*}\right)\left(x-x^{*}\right)+o(t) \\
& =t \nabla H\left(x^{*}\right)\left(x-x^{*}\right)+o(t) \in-K_{3}
\end{aligned}
$$

where $\lim _{t \downarrow 0} o(t)=0$. That is, $H\left(x^{*}+t\left(x-x^{*}\right)\right)=0$.

Therefore $x^{*}+t\left(x-x^{*}\right)$ is feasible for the problem (VPE). Also

$$
\begin{aligned}
& F\left(x^{*}+t\left(x-x^{*}\right)\right)=F\left(x^{*}\right)+t \nabla F\left(x^{*}\right)\left(x-x^{*}\right)+o(t) \\
\Longrightarrow & F\left(x^{*}+t\left(x-x^{*}\right)\right)-F\left(x^{*}\right)=t \nabla F\left(x^{*}\right)\left(x-x^{*}\right)+o(t) \in-\operatorname{int} K_{1}
\end{aligned}
$$

where $\lim _{t \downarrow 0} o(t)=0$. But this contradicts the fact that $x^{*}$ is a weak minimum of the problem (VPE).

Thus by the theorem of alternative given by Illés and Kassay [17], Theorem 3.1, there exist $\alpha^{*} \in K_{1}^{+}, \nu^{*} \in K_{2}^{+}$and $\beta^{*} \in K_{3}^{+}=\mathbb{R}^{l}$ with $\left(\alpha^{*}, \nu^{*}, \beta^{*}\right) \neq 0$ such that

$$
\begin{array}{r}
\left(x-x^{*}\right)^{T}\left[\nabla\left(\alpha^{* T} F\right)\left(x^{*}\right)+\nabla\left(\nu^{* T} G\right)\left(x^{*}\right)+\nabla\left(\beta^{* T} H\right)\left(x^{*}\right)\right]+\left(\nu^{* T} G\right)\left(x^{*}\right) \geq 0 \\
\text { for all } x \in S
\end{array}
$$

For $x=x^{*}$, the above inequality gives

$$
\left(\nu^{* T} G\right)\left(x^{*}\right) \geq 0 .
$$


Since $-G\left(x^{*}\right) \in K_{2}$ and $\nu^{*} \in K_{2}^{+}$, we have

$$
\left(\nu^{* T} G\right)\left(x^{*}\right) \leq 0
$$

Therefore the above two inequalities imply that

$$
\left(\nu^{* T} G\right)\left(x^{*}\right)=0
$$

Also, since $H\left(x^{*}\right)=0$, therefore $\left(\beta^{* T} H\right)\left(x^{*}\right)=0$.

Thus we obtain

$$
\begin{gathered}
\left(x-x^{*}\right)^{T}\left[\nabla\left(\alpha^{* T} F\right)\left(x^{*}\right)+\nabla\left(\nu^{* T} G\right)\left(x^{*}\right)+\nabla\left(\beta^{* T} H\right)\left(x^{*}\right)\right] \geq 0 \text { for all } x \in S \\
\left(\nu^{* T} G\right)\left(x^{*}\right)=0 \text { and }\left(\beta^{* T} H\right)\left(x^{*}\right)=0 .
\end{gathered}
$$

Theorem 4.9 Converse Duality. Let $(\hat{u}, \hat{z}, \hat{w}, \hat{\lambda}, \hat{\mu})$ be a weak maximum of (UD) and suppose the following conditions are satisfied:

(a) $f$ and $g$ are twice differentiable,

(b) $\nabla_{u u}\left(\hat{\lambda}^{T} f\right)(\hat{u})+\nabla_{u u}\left(\hat{\mu}^{T} g\right)(\hat{u})$ is negative definite matrix,

(c) The set $\left\{\nabla f_{i}(\hat{u})+\hat{z} k_{i}: i=1,2, \ldots, m\right\}$ is linearly independent,

(d) $\hat{\mu}^{T} q>0$,

(e) Conditions of Weak Duality Theorem 4.3 or 4.6 are satisfied for each feasible solution $x$ of $(N V P)$.

Then $\hat{u}$ is a weak minimum of (NVP)

Proof. Since $(\hat{u}, \hat{z}, \hat{w}, \hat{\lambda}, \hat{\mu})$ is a weak maximum of (UD), therefore it will be a weak minimum of the following program:

$K$-Minimize $-f(u)-\left(u^{T} z\right) k-\delta\left[\left(\mu^{T} g\right)(u)+u^{T} w\left(\mu^{T} q\right)\right] k$ subject to

$$
\begin{aligned}
& \nabla\left(\lambda^{T} f\right)(u)+z\left(\lambda^{T} k\right)+\nabla\left(\mu^{T} g\right)(u)+w\left(\mu^{T} q\right)=0 \\
& (1-\delta)\left[\left(\mu^{T} g\right)(u)+u^{T} w\left(\mu^{T} q\right)\right] \geq 0 \\
& \delta\left(\lambda^{T} k\right)=\delta .
\end{aligned}
$$

where $u \in \mathbb{R}^{n}, z \in C, w \in D, \lambda \in K^{+} \backslash\{0\}, \mu \in Q^{+}$. 
Therefore by Lemma 4.8 , there exist $\hat{\alpha} \in K^{+}, \hat{\beta} \in \mathbb{R}^{n}, \hat{\xi} \geq 0$ and $\hat{\gamma} \in \mathbb{R}$ with $(\hat{\alpha}, \hat{\beta}, \hat{\xi}, \hat{\gamma}) \neq 0$ such that

$$
\begin{aligned}
& (u-\hat{u})^{T}\left[-\nabla\left(\hat{\alpha}^{T} f\right)(\hat{u})-\left(\hat{\alpha}^{T} k\right) \hat{z}-\delta\left(\hat{\alpha}^{T} k\right)\left\{\nabla\left(\hat{\mu}^{T} g\right)(\hat{u})+\hat{w}\left(\hat{\mu}^{T} q\right)\right\}\right. \\
& \left.-(1-\delta) \hat{\xi}\left\{\nabla\left(\hat{\mu}^{T} g\right)(\hat{u})+\hat{w}\left(\hat{\mu}^{T} q\right)\right\}+\left\{\nabla_{u u}\left(\hat{\lambda}^{T} f\right)(\hat{u})+\nabla_{u u}\left(\hat{\mu}^{T} g\right)(\hat{u})\right\} \hat{\beta}\right] \\
& +(z-\hat{z})^{T}\left[-\left(\hat{\alpha}^{T} k\right) \hat{u}+\left(\hat{\lambda}^{T} k\right) \hat{\beta}\right] \\
& +(w-\hat{w})^{T}\left[-\delta\left(\hat{\alpha}^{T} k\right) \hat{u}-(1-\delta) \hat{\xi} \hat{u}+\hat{\beta}\right]\left(\hat{\mu}^{T} q\right) \\
& +(\lambda-\hat{\lambda})^{T}\left[\nabla f(\hat{u}) \hat{\beta}+\left(\hat{\beta}^{T} \hat{z}\right) k+\delta \hat{\gamma} k\right] \\
& +(\mu-\hat{\mu})^{T}\left[-\delta\left(\hat{\alpha}^{T} k\right)\left\{g(\hat{u})+\left(\hat{u}^{T} \hat{w}\right) q\right\}\right. \\
& \left.-(1-\delta) \hat{\xi}\left\{g(\hat{u})+\left(\hat{u}^{T} \hat{w}\right) q\right\}+\nabla g(\hat{u}) \hat{\beta}+\left(\hat{\beta}^{T} \hat{w}\right) q\right] \geq 0 \\
& \text { for all } u \in \mathbb{R}^{n}, z \in C, w \in D, \lambda \in K^{+} \backslash\{0\}, \mu \in Q^{+} . \\
& \hat{\beta}^{T}\left[\nabla\left(\hat{\lambda}^{T} f\right)(\hat{u})+\hat{z}\left(\hat{\lambda}^{T} k\right)+\nabla\left(\hat{\mu}^{T} g\right)(\hat{u})+\hat{w}\left(\hat{\mu}^{T} q\right)\right]=0 . \\
& (1-\delta) \hat{\xi}\left[\left(\hat{\mu}^{T} g\right)(\hat{u})+\hat{u}^{T} \hat{w}\left(\hat{\mu}^{T} q\right)\right]=0 . \\
& \delta \hat{\gamma}\left[\left(\hat{\lambda}^{T} k\right)-1\right]=0 .
\end{aligned}
$$

Substituting $z=\hat{z}, w=\hat{w}, \lambda=\hat{\lambda}$ and $\mu=\hat{\mu}$ in inequality (4.6), we get

$$
\begin{gathered}
(u-\hat{u})^{T}\left[-\nabla\left(\hat{\alpha}^{T} f\right)(\hat{u})-\left(\hat{\alpha}^{T} k\right) \hat{z}-\delta\left(\hat{\alpha}^{T} k\right)\left\{\nabla\left(\hat{\mu}^{T} g\right)(\hat{u})+\hat{w}\left(\hat{\mu}^{T} q\right)\right\}\right. \\
-(1-\delta) \hat{\xi}\left\{\nabla\left(\hat{\mu}^{T} g\right)(\hat{u})+\hat{w}\left(\hat{\mu}^{T} q\right)\right\} \\
\left.+\left\{\nabla_{u u}\left(\hat{\lambda}^{T} f\right)(\hat{u})+\nabla_{u u}\left(\hat{\mu}^{T} g\right)(\hat{u})\right\} \hat{\beta}\right] \geq 0 \quad \text { for all } u \in \mathbb{R}^{n}
\end{gathered}
$$

and proceeding the similar manner we get the following inequalities

$$
\begin{gathered}
(z-\hat{z})^{T}\left[-\left(\hat{\alpha}^{T} k\right) \hat{u}+\left(\hat{\lambda}^{T} k\right) \hat{\beta}\right] \geq 0 \quad \text { for all } z \in C \\
(w-\hat{w})^{T}\left[-\delta\left(\hat{\alpha}^{T} k\right) \hat{u}-(1-\delta) \hat{\xi} \hat{u}+\hat{\beta}\right]\left(\hat{\mu}^{T} q\right) \geq 0 \quad \text { for all } w \in D \\
(\lambda-\hat{\lambda})^{T}\left[\nabla f(\hat{u}) \hat{\beta}+\left(\hat{\beta}^{T} \hat{z}\right) k+\delta \hat{\gamma} k\right] \geq 0 \quad \text { for all } \lambda \in K^{+} \backslash\{0\} \\
(\mu-\hat{\mu})^{T}\left[-\delta\left(\hat{\alpha}^{T} k\right)\left\{g(\hat{u})+\left(\hat{u}^{T} \hat{w}\right) q\right\}-(1-\delta) \hat{\xi}\left\{g(\hat{u})+\left(\hat{u}^{T} \hat{w}\right) q\right\}\right. \\
\left.+\nabla g(\hat{u}) \hat{\beta}+\left(\hat{\beta}^{T} \hat{w}\right) q\right] \geq 0 \quad \text { for all } \mu \in Q^{+} .
\end{gathered}
$$

It must be noted here that if the value of $\delta=0$, then we would not have the third constraint $\delta\left(\lambda^{T} k\right)=\delta$ in our dual problem (UD) and hence the multiplier 
$\hat{\gamma}$ will not come into existence and similarly if $\delta=1$ then the multiplier $\hat{\xi}$ will not come into existence.

Inequality (4.7) can be written as,

$$
\begin{aligned}
-\nabla\left(\hat{\alpha}^{T} f\right)(\hat{u})-\left(\hat{\alpha}^{T} k\right) \hat{z}-\left\{\delta\left(\hat{\alpha}^{T} k\right)\right. & +(1-\delta) \hat{\xi}\}\left\{\nabla\left(\hat{\mu}^{T} g\right)(\hat{u})+\hat{w}\left(\hat{\mu}^{T} q\right)\right\} \\
& +\left\{\nabla_{u u}\left(\hat{\lambda}^{T} f\right)(\hat{u})+\nabla_{u u}\left(\hat{\mu}^{T} g\right)(\hat{u})\right\} \hat{\beta}=0,
\end{aligned}
$$

which implies that

$$
\begin{aligned}
& \left\{\nabla_{u u}\left(\hat{\lambda}^{T} f\right)(\hat{u})+\nabla_{u u}\left(\hat{\mu}^{T} g\right)(\hat{u})\right\} \hat{\beta} \\
& \quad=\nabla\left(\hat{\alpha}^{T} f\right)(\hat{u})+\left(\hat{\alpha}^{T} k\right) \hat{z}+\left\{\delta\left(\hat{\alpha}^{T} k\right)+(1-\delta) \hat{\xi}\right\}\left\{\nabla\left(\hat{\mu}^{T} g\right)(\hat{u})+\hat{w}\left(\hat{\mu}^{T} q\right)\right\} .
\end{aligned}
$$

On using the feasibility of $(\hat{u}, \hat{z}, \hat{w}, \hat{\lambda}, \hat{\mu})$ for (UD) in above equality, we get

$$
\begin{aligned}
& \left\{\nabla_{u u}\left(\hat{\lambda}^{T} f\right)(\hat{u})+\nabla_{u u}\left(\hat{\mu}^{T} g\right)(\hat{u})\right\} \hat{\beta} \\
& =\nabla\left(\hat{\alpha}^{T} f\right)(\hat{u})+\left(\hat{\alpha}^{T} k\right) \hat{z}-\left\{\delta\left(\hat{\alpha}^{T} k\right)+(1-\delta) \hat{\xi}\right\}\left\{\nabla\left(\hat{\lambda}^{T} f\right)(\hat{u})+\hat{z}\left(\hat{\lambda}^{T} k\right)\right\} \\
& =\sum_{i=1}^{m}\left\{\hat{\alpha}_{i}-\left(\delta\left(\hat{\alpha}^{T} k\right)+(1-\delta) \hat{\xi}\right) \hat{\lambda}_{i}\right\}\left\{\nabla f_{i}(\hat{u})+\hat{z} k_{i}\right\} .
\end{aligned}
$$

Premultiplying the above equation with $\hat{\beta}^{T}$, we get

$$
\begin{aligned}
\hat{\beta}^{T}\left\{\nabla_{u u}\left(\hat{\lambda}^{T} f\right)(\hat{u})\right. & \left.+\nabla_{u u}\left(\hat{\mu}^{T} g\right)(\hat{u})\right\} \hat{\beta} \\
= & \hat{\beta}^{T} \sum_{i=1}^{m}\left\{\hat{\alpha}_{i}-\delta\left(\hat{\alpha}^{T} k\right) \hat{\lambda}_{i}\right\}\left\{\nabla f_{i}(\hat{u})+\hat{z} k_{i}\right\} \\
& \quad(1-\delta) \hat{\xi} \hat{\beta}^{T}\left\{\nabla\left(\hat{\lambda}^{T} f\right)(\hat{u})+\hat{z}\left(\hat{\lambda}^{T} k\right)\right\} .
\end{aligned}
$$

Substituting $\lambda=\frac{\hat{\lambda}}{2}$ and $\lambda=\frac{3 \hat{\lambda}}{2}$ in relation (4.10) and using that $\delta\left(\hat{\lambda}^{T} k\right)=\delta$, we get

$$
\hat{\beta}^{T} \nabla\left(\hat{\lambda}^{T} f\right)(\hat{u})+\hat{\beta}^{T} \hat{z}\left(\hat{\lambda}^{T} k\right)=-\delta \hat{\gamma} .
$$

From (4.13) and (4.14), we get

$$
\begin{aligned}
\hat{\beta}^{T}\left\{\nabla_{u u}\left(\hat{\lambda}^{T} f\right)(\hat{u})+\nabla_{u u}\right. & \left.\left(\hat{\mu}^{T} g\right)(\hat{u})\right\} \hat{\beta} \\
& =\hat{\beta}^{T} \sum_{i=1}^{m}\left\{\hat{\alpha}_{i}-\delta\left(\hat{\alpha}^{T} k\right) \hat{\lambda}_{i}\right\}\left\{\nabla f_{i}(\hat{u})+\hat{z} k_{i}\right\}-(1-\delta) \delta \hat{\xi} \hat{\gamma} .
\end{aligned}
$$


Since $\delta \in\{0,1\}$, therefore the above equation reduces to

$$
\hat{\beta}^{T}\left\{\nabla_{u u}\left(\hat{\lambda}^{T} f\right)(\hat{u})+\nabla_{u u}\left(\hat{\mu}^{T} g\right)(\hat{u})\right\} \hat{\beta}=\hat{\beta}^{T} \sum_{i=1}^{m}\left\{\hat{\alpha}_{i}-\delta\left(\hat{\alpha}^{T} k\right) \hat{\lambda}_{i}\right\}\left\{\nabla f_{i}(\hat{u})+\hat{z} k_{i}\right\} .
$$

First we will prove that $\hat{\alpha} \neq 0$. For if $\hat{\alpha}=0$, then by equation (4.15) we have

$$
\hat{\beta}^{T}\left\{\nabla_{u u}\left(\hat{\lambda}^{T} f\right)(\hat{u})+\nabla_{u u}\left(\hat{\mu}^{T} g\right)(\hat{u})\right\} \hat{\beta}=0
$$

which on using condition (b) of this theorem gives $\hat{\beta}=0$.

By substituting $\hat{\beta}=0$ in equation (4.14) we have

$$
\delta \hat{\gamma}=0
$$

As mentioned earlier if $\delta=0$, then $\hat{\gamma}$ will not come into existence and if $\delta=1$, then from above equation $\hat{\gamma}=0$. Substituting $\hat{\alpha}=0$ and $\hat{\beta}=0$ in equation (4.12) we have

$$
\sum_{i=1}^{m}(1-\delta) \hat{\xi} \hat{\lambda}_{i}\left\{\nabla f_{i}(\hat{u})+\hat{z} k_{i}\right\}=0
$$

which on using condition (c) implies that for each $i=1,2, \ldots, m, \hat{\xi}(1-\delta) \hat{\lambda}_{i}=0$. But since $\hat{\lambda} \in K^{+} \backslash\{0\}$, therefore we have

$$
\hat{\xi}(1-\delta)=0
$$

Again if $\delta=1$, then $\hat{\xi}$, will not come into picture and if $\delta=0$, then from above equation $\hat{\xi}=0$.

We finally arrive at a condition where $\hat{\alpha}=0, \hat{\beta}=0, \hat{\xi}=0, \hat{\gamma}=0$, which a contradiction to the fact that $(\hat{\alpha}, \hat{\beta}, \hat{\xi}, \hat{\gamma}) \neq 0$. Hence $\hat{\alpha} \neq 0$.

Since $\hat{\alpha} \in K^{+} \backslash\{0\}$ and $k \in \operatorname{int} K$, therefore $\hat{\alpha}^{T} k>0$ and hence $\frac{1}{\left(\hat{\alpha}^{T} k\right)} \hat{\alpha} \in$ $K^{+} \backslash\{0\}$. Now substituting $\lambda=\frac{1}{\left(\hat{\alpha}^{T} k\right)} \hat{\alpha}$ in (4.10), we have

$$
\left(\hat{\alpha}-\left(\hat{\alpha}^{T} k\right) \hat{\lambda}\right)^{T}\left[\nabla f(\hat{u}) \hat{\beta}+\left(\hat{\beta}^{T} \hat{z}\right) k+\delta \hat{\gamma} k\right] \geq 0
$$

Using the fact that $\delta\left(\hat{\lambda}^{T} k\right)=\delta$, we get

$$
\left(\hat{\alpha}-\left(\hat{\alpha}^{T} k\right) \hat{\lambda}\right)^{T}\left[\nabla f(\hat{u}) \hat{\beta}+k\left(\hat{\beta}^{T} \hat{z}\right)\right] \geq 0
$$

which gives that

$$
\hat{\beta}^{T}\left[\nabla\left(\hat{\alpha}^{T} f\right)(\hat{u})+\left(\hat{\alpha}^{T} k\right) \hat{z}\right] \geq\left(\hat{\alpha}^{T} k\right) \hat{\beta}^{T}\left[\nabla\left(\hat{\lambda}^{T} f\right)(\hat{u})+\left(\hat{\lambda}^{T} k\right) \hat{z}\right]
$$


or

$$
\hat{\beta}^{T} \sum_{i=1}^{m} \hat{\alpha}_{i}\left\{\nabla f_{i}(\hat{u})+\hat{z} k_{i}\right\} \geq \hat{\beta}^{T} \sum_{i=1}^{m}\left(\hat{\alpha}^{T} k\right) \hat{\lambda}_{i}\left\{\nabla f_{i}(\hat{u})+\hat{z} k_{i}\right\} .
$$

Substituting the above inequality in (4.15), we get

$$
\hat{\beta}^{T}\left\{\nabla_{u u}\left(\hat{\lambda}^{T} f\right)(\hat{u})+\nabla_{u u}\left(\hat{\mu}^{T} g\right)(\hat{u})\right\} \hat{\beta} \geq \hat{\beta}^{T} \sum_{i=1}^{m}(1-\delta)\left(\hat{\alpha}^{T} k\right) \hat{\lambda}_{i}\left\{\nabla f_{i}(\hat{u})+\hat{z} k_{i}\right\}
$$

or

$$
\begin{aligned}
\hat{\beta}^{T}\left\{\nabla_{u u}\left(\hat{\lambda}^{T} f\right)(\hat{u})\right. & \left.+\nabla_{u u}\left(\hat{\mu}^{T} g\right)(\hat{u})\right\} \hat{\beta} \\
& \geq(1-\delta)\left(\hat{\alpha}^{T} k\right) \hat{\beta}^{T}\left[\nabla\left(\hat{\lambda}^{T} f\right)(\hat{u})+\left(\hat{\lambda}^{T} k\right) \hat{z}\right] .
\end{aligned}
$$

Now using (4.14) in above inequality, we have

$$
\hat{\beta}^{T}\left\{\nabla_{u u}\left(\hat{\lambda}^{T} f\right)(\hat{u})+\nabla_{u u}\left(\hat{\mu}^{T} g\right)(\hat{u})\right\} \hat{\beta} \geq-\delta(1-\delta) \hat{\gamma}\left(\hat{\alpha}^{T} k\right),
$$

and since $\delta \in\{0,1\}$, we have

$$
\hat{\beta}^{T}\left\{\nabla_{u u}\left(\hat{\lambda}^{T} f\right)(\hat{u})+\nabla_{u u}\left(\hat{\mu}^{T} g\right)(\hat{u})\right\} \hat{\beta} \geq 0 .
$$

On using condition (b), we get $\hat{\beta}=0$.

Putting the value $\hat{\beta}=0$ in (4.12), we get

$$
\sum_{i=1}^{m}\left\{\hat{\alpha}_{i}-\left(\delta\left(\hat{\alpha}^{T} k\right)+(1-\delta) \hat{\xi}\right) \hat{\lambda}_{i}\right\}\left\{\nabla f_{i}(\hat{u})+\hat{z} k_{i}\right\}=0
$$

which on using condition (c), gives

$$
\hat{\alpha}_{i}=\left\{\left(\hat{\alpha}^{T} k\right) \delta+\hat{\xi}(1-\delta)\right\} \hat{\lambda}_{i}, \text { for each } i=1,2, \ldots, m
$$

That is, for each $i=1,2, \ldots, m$,

$$
\hat{\alpha}_{i}= \begin{cases}\hat{\xi} \hat{\lambda}_{i} ; & \text { if } \delta=0 \\ \left(\hat{\alpha}^{T} k\right) \hat{\lambda}_{i} ; & \text { if } \delta=1\end{cases}
$$

Since $\hat{\alpha}, \hat{\lambda} \in K^{+} \backslash\{0\}$, so if $\delta=0$, then in that case we have $\hat{\xi}>0$.

Putting the value $\hat{\beta}=0$ in (4.8) and using the fact that $\hat{\alpha}^{T} k>0$, we get

$$
\hat{u}^{T} z \leq \hat{u}^{T} \hat{z} \quad \text { for all } z \in C,
$$

which implies that

$$
\hat{u}^{T} \hat{z}=\max \left\{\hat{u}^{T} z: z \in C\right\}=s(\hat{u} \mid C), \quad \text { that is } \hat{z} \in \partial s(\hat{u} \mid C) .
$$


Similarly substituting $\hat{\beta}=0$ in (4.9), we get

$$
-(w-\hat{w})^{T}\left\{\delta\left(\hat{\alpha}^{T} k\right) \hat{u}+(1-\delta) \hat{\xi}\right\}\left(\hat{\mu}^{T} q\right) \geq 0 \quad \text { for all } w \in D
$$

which on using the fact that $\delta \in\{0,1\}, \hat{\alpha}^{T} k>0, \hat{\xi}>0$ and $\hat{\mu}^{T} q>0$, becomes

$$
\hat{u}^{T} w \leq \hat{u}^{T} \hat{w} \quad \text { for all } w \in D,
$$

which implies that

$$
\hat{u}^{T} \hat{w}=\max \left\{\hat{u}^{T} w: w \in D\right\}=s(\hat{u} \mid D), \quad \text { that is } \hat{w} \in \partial s(\hat{u} \mid D) .
$$

Substituting $\hat{\beta}=0$ in (4.11) and using the fact that $\delta \in\{0,1\}, \hat{\alpha}^{T} k>0$ and $\hat{\xi}>0$, we have

$$
-(\mu-\hat{\mu})^{T}\left[g(\hat{u})+\left(\hat{u}^{T} \hat{w}\right) q\right] \geq 0 \quad \text { for all } \mu \in Q^{+} .
$$

Since $\mu, \hat{\mu} \in Q^{+}$and $Q^{+}$is a closed convex cone therefore replacing $\mu$ by $\mu+\hat{\mu}$ and using $\hat{u}^{T} \hat{w}=s(\hat{u} \mid D)$ in the above inequality, we get

$$
-\mu^{T}[g(\hat{u})+s(\hat{u} \mid D) q] \geq 0 \quad \text { for all } \mu \in Q^{+},
$$

which implies that

$$
-g(\hat{u})-s(\hat{u} \mid D) q \in Q
$$

Hence $\hat{u}$ is feasible for (NVP).

Since $(\hat{u}, \hat{z}, \hat{w}, \hat{\lambda}, \hat{\mu})$ is feasible for (UD) and conditions of Weak Duality result 4.3 or 4.6 are satisfied for each feasible solution $x$ of (NVP), we have for each $x \in S_{0}$,

$$
f(u)+\left(u^{T} z\right) k+\delta\left[\left(\mu^{T} g\right)(u)+u^{T} w\left(\mu^{T} q\right)\right] k-f(x)-s(x \mid C) k \notin \operatorname{int} K .
$$

Substituting $\mu=0$ and $\mu=2 \hat{\mu}$ in (4.16), we get

$$
\left(\hat{\mu}^{T} g\right)(\hat{u})+\hat{u}^{T} \hat{w}\left(\hat{\mu}^{T} q\right)=0 .
$$

Using $\hat{u}^{T} \hat{z}=s(\hat{u} \mid C)$ and above equation in (4.17), we have

$$
f(\hat{u})+s(\hat{u} \mid C) k-f(x)-s(x \mid C) k \notin \operatorname{int} K .
$$

Therefore, $\hat{u}$ is weak minimum for (NVP).

\section{Special Cases}

In this section we specialize our problem.

1. Let the compact sets $C$ and $D$ be

$$
C=\left\{A z: z^{T} A z \leq 1\right\} \text { and } D=\left\{B w: w^{T} B w \leq 1\right\}
$$


where $A$ and $B$ are positive semidefinite matrices of order $n$. As discussed by Mond and Schechter [2], we may write

$$
s(x \mid C)=\left(x^{T} A x\right)^{1 / 2} \text { and } s(x \mid D)=\left(x^{T} B x\right)^{1 / 2} .
$$

Putting these values in our problems, we get

$(\mathrm{NVP})_{1} \quad K$-Minimize $f(x)+\left(x^{T} A x\right)^{1 / 2} k$

subject to

$$
-g(x)-\left(x^{T} B x\right)^{1 / 2} q \in Q,
$$

Similarly substituting these values in dual problem, we get

$$
\begin{aligned}
& \text { K-Maximize } f(u)+\left(u^{T} A z\right) k+\delta\left[\left(\mu^{T} g\right)(u)+u^{T} B w\left(\mu^{T} q\right)\right] k \\
& \text { subject to } \\
& \qquad \nabla\left(\lambda^{T} f\right)(u)+A z\left(\lambda^{T} k\right)+\nabla\left(\mu^{T} g\right)(u)+B w\left(\mu^{T} q\right)=0 \\
& \quad(1-\delta)\left[\left(\mu^{T} g\right)(u)+u^{T} B w\left(\mu^{T} q\right)\right] \geq 0 \\
& \quad \delta\left(\lambda^{T} k\right)=\delta .
\end{aligned}
$$

If we take $m=p$ and $p=q$ and replace $g_{i}$ by the function $G_{i}$, also if we take $K=\mathbb{R}_{+}^{p}, Q=\mathbb{R}_{+}^{q}, k=(1,1, \ldots, 1) \in \mathbb{R}^{p}$ and $q=(1,1, \ldots, 1) \in \mathbb{R}^{q}$. Then our problem $(\mathrm{NVP})_{1}$ reduces to the problem $(P 5)$ considered by Zalmai [7] where $X=\mathbb{R}^{n}$, the set $\underline{r}$ is empty, $P_{i}=A$ for each $i=1,2, \ldots, p$ and $R_{j}=B$ for each $j=1,2, \ldots, q$.

If we interchange the roles of $m$ and $p$ and take $K=\mathbb{R}_{+}^{p}, Q=\mathbb{R}_{+}^{m}, k=$ $(1,1, \ldots, 1) \in \mathbb{R}^{p}$ and $q=(1,1, \ldots, 1) \in \mathbb{R}^{m}$, then our problem $(\mathrm{NVP})_{1}$ reduces to $(\mathrm{MNP})_{1}$ and the unified dual $(\mathrm{UD})_{1}$ reduces to $(\mathrm{WND})_{1}$ and $(\mathrm{M}-\mathrm{WND})_{1}$ if $\delta=1$ and $\delta=0$, respectively, considered by Husain et al. [8] where $B^{i}=A$ for each $i=1,2, \ldots, p$ and $E^{j}=B$ for each $j=1,2, \ldots, m$.

2. Let the compact convex set $C$ and $D$ be

$$
\begin{gathered}
C=\left\{A z:\|z\|_{p_{1}} \leq 1\right\} \\
D=\left\{B w:\|w\|_{p_{1}} \leq 1\right\},
\end{gathered}
$$

where $A$ and $B$ are $n \times n$ matrices. As shown by Mond and Schechter [2], we can write

$$
s(x \mid C)=\|A x\|_{p_{2}} \quad s(x \mid D)=\|B x\|_{p_{2}}
$$

where $p_{1}$ and $p_{2}$ are conjugate exponents, i. e., for $p_{2}>1, \frac{1}{p_{1}}+\frac{1}{p_{2}}=1$.

Then substituting these values of $s(x \mid C)$ and $s(x \mid D)$ in the problem $(N V P)$, we get

$(\mathrm{NVP})_{2} \quad K$-Minimize $f(x)+\|A x\|_{p_{2}} k$ subject to 


$$
-g(x)-\|B x\|_{p_{2}} q \in Q,
$$

and its unified dual is given as under

$(\mathrm{UD})_{2} \quad K$-Maximize $f(u)+\left(u^{T} A z\right) k+\delta\left[\left(\mu^{T} g\right)(u)+u^{T} B w\left(\mu^{T} q\right)\right] k$ subject to

$$
\begin{aligned}
& \nabla\left(\lambda^{T} f\right)(u)+A z\left(\lambda^{T} k\right)+\nabla\left(\mu^{T} g\right)(u)+B w\left(\mu^{T} q\right)=0 \\
& (1-\delta)\left[\left(\mu^{T} g\right)(u)+u^{T} B w\left(\mu^{T} q\right)\right] \geq 0 \\
& \delta\left(\lambda^{T} k\right)=\delta . \\
\text { where } & u, z, w \in \mathbb{R}^{n},\|z\|_{p_{1}} \leq 1,\|w\|_{p_{1}} \leq 1, \lambda \in K^{+} \backslash\{0\}, \mu \in Q^{+} .
\end{aligned}
$$

If we take $m=p$ and $p=q$ and replace $g_{i}$ by the function $G_{i}$, also if we take $K=\mathbb{R}_{+}^{p}, Q=\mathbb{R}_{+}^{q}, k=(1,1, \ldots, 1) \in \mathbb{R}^{p}$ and $q=(1,1, \ldots, 1) \in \mathbb{R}^{q}$. Then our problem $(\mathrm{NVP})_{2}$ reduces to the problem $(P 1)$ considered by Zalmai [7] where $X=\mathbb{R}^{n}$, the set $\underline{r}$ is empty, $A_{i}=A, a(i)=p_{2}$ for each $i=1,2, \ldots, p$ and $C_{j}=B, c_{j}=p_{1}$ for each $j=1,2, \ldots, q$.

If we take $m=1, p=m, K=\mathbf{R}_{+}, k=1, Q=\mathbf{R}_{+}^{m}$ and $q=(1,1, \ldots, 1)^{T} \in Q$ then our Problem (NVP) $)_{1}$ reduces to the problem $(\mathrm{NP})_{1}$ and the dual problem $(\mathrm{UD})_{1}$ reduces to $(\mathrm{WD})_{1}$ and $(\mathrm{M}-\mathrm{WD})_{1}$ if $\delta=1$ and $\delta=0$, respectively, considered by Husain et al. [9] as special case where $B=A$ and $E_{j}=B$ for $j=1,2, \ldots, m$. Further our problem $(\mathrm{NVP})_{2}$ reduces to $(\mathrm{NP})_{2}$ and the unified dual (UD) $)_{2}$ reduces to the problem (WD) $)_{2}$ and (M-WD) $)_{2}$, if $\delta=1$ and $\delta=0$, respectively, where $p=p_{2}, q=p_{1}, P=A$ and $Q_{j}=B$ for $j=1,2, \ldots, m$.

\section{Conclusions}

The main advantage of this paper is that the problem (NVP) considered here generalises many existing problems in the literature. As mentioned in Remark 3.1, under particular conditions, our problem (NVP) reduces to the problems considered by Schechter [11], Husain et al. [9] and Husain et al. [8]. Also the main result of the paper, Theorem 3.4, is proved without using any convexity assumptions. Moreover, the unified dual (UD), provides a common platform to study both Wolfe type as well as Mond-Weir type duals.

Acknowledgements. The first author is grateful to the University Grants Commission (UGC), India for offering financial support. The second author is thankful to Council of Scientific \& Industrial Research (CSIR), India for the financial support provided during the work. Both the authors are also grateful to the two referees for their valuable suggestions in improving the presentation of the paper.

\section{REFERENCES}

[1] B.D. Craven, Nonsmooth multiobjective programming. Numer. Func. Anal. Optim. 10 (1989) 49-64.

[2] B. Mond and M. Schechter, A duality theorem for a homogeneous fractional programming problem. J. Optim. Theory. Appl. 25 (1978) 349-359. 
[3] D.T. Luc, Theory of vector optimization. Springer (1989).

[4] F. Flores-Bazán and C. Vera, Unifying efficiency and weak efficiency in generalized quasiconvex vector minimization on the real-line. Int. J. Optim. Theory: Theory, Methods and Appl. 1 (2009) 247-265.

[5] F. Flores-Bazán, N. Hadjisavvas and C. Vera, An optimal altenative theorem and applications to mathematical programming. J. Glob. Optim. 37 (2007) 229-243.

[6] F.H. Clarke, Optimization and nonsmooth analysis. A Wiley-Interscience Publication (1983).

[7] G.J. Zalmai, Generalized ( $\eta, \rho)$-invex functions and global semiparametric sufficient efficiency conditions for multiobjective fractional programming problems containing arbitrary norms. J. Glob. Optim. 36 (2006) 51-85.

[8] I. Husain, A. Ahmed and R.G. Mattoo, On multiobjective nonlinear programming with support functions. J. Appl. Anal. 16 (2010) 171-187.

[9] I. Husain, Abha and Z. Jabeen, On nonlinear programming with support functions. J. Appl. Math. Comput. 10 (2002) 83-99.

[10] J. Jahn, Vector optimization: Theory, applications and extensions. Springer (2011).

[11] M. Schechter, A subgradient duality theorem. J. Math. Anal. Appl. 61 (1977) 850-855.

[12] M. Schechter, More on subgradient duality. J. Math. Anal. Appl. 71 (1979) 251-262.

[13] R. Cambini, Some new classes of generalized concave vector-valued functions. Optim. 36 (1996) 11-24.

[14] R. Cambini and L. Carosi, Mixed type duality for multiobjective optimization problems with set constraints, in Optimality conditions in vector optimization, edited by Manuel Arana Jiménez, G. Ruiz-Garzón and A. Rufián-Lizan., Bentham Sci. Publishers, The Netherlands (2010) 119-142.

[15] S.K. Suneja, P. Louhan and M.B. Grover, Higher-order cone-pseudoconvex, quasiconvex and other related functions in vector optimization. Optim. Lett. 7 (2013) 647-664.

[16] S.K. Suneja, S. Sharma and Vani, Second-order duality in vector optimization over cones. J. Appl. Math. Inform. 26 (2008) 251-261.

[17] T. Illés and G. Kassay, Theorems of the alternative and optimality conditions for convexlike and general convexlike programming. J. Optim. Theory. Appl. 101 (1999) 243-257.

[18] T. Weir, B. Mond and B.D. Craven, Weak minimization and duality. Numer. Funct. Anal. Optim. 9 (1987) 181-192. 